TITLE:

\title{
L Discretization for Sampled-Data Controller Synthesis via Piecewise Linear Approximation
}

$\operatorname{AUTHOR}(\mathrm{S})$ :

Kim, Jung Hoon; Hagiwara, Tomomichi

\section{CITATION:}

Kim, Jung Hoon ...[et al]. L Discretization for Sampled-Data Controller Synthesis via Piecewise Linear Approximation. IEEE Transactions on Automatic Control 2016, 61(5): 1143-1157

ISSUE DATE:

2016-05

URL:

http://hdl.handle.net/2433/235594

\section{RIGHT:}

(C) 2016 IEEE. Personal use of this material is permitted. Permission from IEEE must be obtained for all other uses, in any current or future media, including reprinting/republishing this material for advertising or promotional purposes, creating new collective works, for resale or redistribution to servers or lists, or reuse of any copyrighted component of this work in other works.; この論文は出版社版でありません。引用の際には出版社版をご確認ご利用ください。;This is not the published version. Please cite only the published version. 


\title{
$L_{1}$ Discretization for Sampled-Data Controller Synthesis via Piecewise Linear Approximation
}

\author{
Jung Hoon Kim and Tomomichi Hagiwara, Senior Member, IEEE
}

\begin{abstract}
This paper develops a new discretization method with piecewise linear approximation for the $L_{1}$ optimal controller synthesis problem of sampled-data systems, which is the problem of minimizing the $L_{\infty}$-induced norm of sampled-data systems. We apply fast-lifting on the top of the lifting technique, by which the sampling interval $[0, h)$ is divided into $M$ subintervals with an equal width. The signals on each subinterval are then approximated by linear functions by introducing two types of 'linearizing operators' for input and output, which leads to piecewise linear approximation of sampled-data systems. By using the arguments of preadjoint operators, we provide an important inequality that forms a theoretical basis for tackling the $L_{1}$ optimal controller synthesis problem of sampled-data systems more efficiently than the conventional method. More precisely, a mathematical basis for the piecewise linear approximation method associated with the convergence rate is shown through this inequality, and this suggests that the piecewise linear approximation method may drastically outperform the conventional method in the $L_{1}$ optimal controller synthesis problem of sampled-data systems. We then provide a discretization procedure of sampled-data systems by which the $L_{1}$ optimal controller synthesis problem is converted to the discrete-time $l_{1}$ optimal controller synthesis problem. Finally, effectiveness of the proposed method is demonstrated through a numerical example.
\end{abstract}

\section{INTRODUCTION}

The disturbance rejection problem is one of the main issues in control, and system norms are used to evaluate the effect of disturbances. Depending on the nature of the disturbance affecting the system and performance measures, one can define a number of different system norms. Among various system norms, the $\mathrm{H}_{2}$ norm evaluates the power of the output for a white noise disturbance, while the $H_{\infty}$ norm is used to evaluate the energy of the output for the worst disturbances among those with finite energy. However, they cannot be used for dealing with the problems of suppressing the peak amplitude of the output for bounded persistent disturbances, such as steps and sinusoids, which are often encountered in control systems. Instead, the $L_{\infty}$-induced (or $l_{\infty}$-induced) norm should be considered to deal with such peak amplitude.

Let us first review the studies relevant to such induced norms. Because this norm corresponds to the $L_{1}$ (or $l_{1}$ ) norm of the impulse response of the system in the continuous-time

This work was supported in part by JSPS KAKENHI Grant Numbers 14J05353, 24560545 and 15K06138.

J. H. Kim was with the Department of Electrical Engineering, Kyoto University, Kyoto, Japan. He is now with the Center for Robotics Research, Korea Institute of Science and Technology (KIST), 5, Hwarang-ro 14-gil, Seongbukgu, Seoul, 136-791, Republic of Korea (e-mail: kimjunghoon28@gmail.com).

T. Hagiwara is with the Department of Electrical Engineering, Kyoto University, Nishikyo-ku, Kyoto, 615-8510, Japan (e-mail: hagiwara@kuee.kyotou.ac.jp). (or discrete-time) case, the study associated with the treatment of the $L_{\infty}$-induced norm (or $l_{\infty}$-induced norm) has been named the $L_{1}$ (or $l_{1}$ ) problem. The $L_{1}$ (or $l_{1}$ ) optimal control deals with the design of optimal controllers that minimizes the peak amplitude of the output caused by unknown bounded persistent disturbances, and this control objective matches practical applications such as avoiding mechanical systems from colliding with their surrounding objects and protecting chemical systems from being overly pressured, for which the $H_{2}$ and $H_{\infty}$ control objectives do not match effectively. A more detailed summary of relevant studies is as follows.

Some special cases of $L_{1}$ (or $l_{1}$ ) control problem have been formulated but a general case was not dealt with in [1]. The general case of the continuous-time $L_{1}$ problem was discussed in [2], [3] while the discrete-time $l_{1}$ problem was dealt with in [4]-[7]. Stimulated by the success in these studies, the $L_{1}$ problem of sampled-data systems (with intersample behavior taken into account) has been studied in [8]-[10]. However, in contrast to the studies relevant to modeling [11], [12], the $H_{2}$ problem [13]-[18] and the $H_{\infty}$ problem [17][26] of sampled-data systems ${ }^{1}$, no exact solution has been obtained for the $L_{1}$ problem of sampled-data systems and only approximate methods have been provided. More precisely, in [8]-[10], a sampled-data system is approximately treated as a discrete-time system through the fast-sample/fast-hold (FSFH) approximation technique [29], and it is shown that the $l_{\infty}$-induced norm of the resulting discrete-time system converges to the $L_{\infty}$-induced norm of the original sampleddata system at the rate of $1 / M$, as the FSFH approximation parameter $M$ tends to infinity.

Next, let us review our recent studies relevant to the $L_{1}$ problem of sampled-data systems. As a significant advance over the conventional methods through the FSFH approximation, the present authors developed in [30] an extended method for the $L_{1}$ problem of sampled-data systems by using the ideas of fast-lifting [25] and piecewise linear approximation [31]. Fast-lifting also has an integer parameter $M$ as in the FSFH approximation technique. However, it is used only to subdivide the sampling interval $[0, h)$ into $M$ smaller pieces, while the conventional FSFH approximation technique takes $M$ equally spaced sampling points on the interval $[0, h)$; no information is hence lost as to signals on $[0, h)$ by fast-lifting. This feature

\footnotetext{
${ }^{1}$ We note that the study in [27] plays a significant role in the $H_{\infty}$ problem with respect to the necessary condition or the so-called $\gamma$-contractiveness of a compression operator; it would be worthwhile to remark that the importance of this study further stimulated another study in [28], which is also very closely related to the treatment of this contractiveness condition pertinent to the $H_{\infty}$ problem.
} 
is crucial in developing a piecewise linear approximation technique, while the FSFH approximation technique corresponds to applying only piecewise constant approximation. A method for computing an upper bound and a lower bound of the $L_{\infty}$-induced norm of sampled-data systems through piecewise linear approximation is provided and it is shown that the gap between the upper and lower bounds converges to 0 at convergence rate $1 / M^{2}$ for the fast-lifting parameter $M$. Unfortunately, however, this method is restricted to analysis and cannot be used directly for synthesis. This is because it requires to compute the $L_{1}[0, h / M)$ norms of linear kernel functions determined by the continuous-time generalized plant and the discrete-time controller and the structure of the way the controller parameters are involved in the linear kernel functions is complicated.

In contrast, the present paper aims at establishing a discretization procedure of the generalized plant for the $L_{1}$ optimal controller synthesis problem of sampled-data systems via piecewise linear approximation. This approximation is achieved by introducing two types of 'linearizing operators' for signals on the interval $[0, h / M)$ obtained by applying fastlifting, one for the input signals and the other for the output signals. By applying the arguments of preadjoint operators, we provide an important inequality that forms a theoretical basis for the piecewise linear approximation approach to the $L_{1}$ optimal controller synthesis problem, together with a convergence proof again in the rate of $1 / M^{2}$ and derivation of associated error bounds. In connection with this improved convergence rate, we further give a discretization procedure of the generalized plant for the sampled-data $L_{1}$ optimal controller synthesis through the piecewise linear approximation treatment.

The organization of this paper is as follows. In Section II, we give some mathematical preliminaries. We then review in Section III the lifted representation of sampled-data systems [21] [23] to take into account of their intersample behavior. In Section IV, we introduce two 'linearizing operators' and provide a piecewise linear approximation procedure of sampled-data systems. Section $\mathrm{V}$ provides one of the main arguments of this paper, in which we carry out an error analysis and provide a solid mathematical basis associated with the piecewise linear approximation treatment; the mathematical basis enables us to tackle the $L_{1}$ optimal controller synthesis problem through the piecewise linear approximation treatment and shows the advantage of such an approach over the existing method based on the FSFH approximation treatment. In Section VI, we give the second main arguments of this paper, in which a discretization method for the generalized plant is provided to solve the $L_{1}$ optimal controller synthesis problem of sampleddata systems through piecewise linear approximation. We examine effectiveness of our piecewise linear approximation method through a numerical example in Section VII. We state concluding remarks in Section VIII.

\section{Mathematical PReliminaries}

This section gives some mathematical preliminaries. We use the notations $\mathbb{N}, \mathbb{R}_{\infty}^{\nu}, \mathbb{R}_{1}^{\nu}$ and $R(\cdot)$ to denote the the set of positive integers, the Banach space of $\nu$-dimensional real vectors equipped with vector $\infty$-norm, the Banach space of $\nu$-dimensional real vectors equipped with vector 1 -norm, and the range of an operator, respectively.

The dual space of a Banach space $X$, i.e., the space of all bounded linear functionals on $X$, is denoted by $X^{*}$.

Let $X$ and $Y$ be Banach spaces. For a linear operator $\mathbf{T}$ : $X \rightarrow Y$, its adjoint [32]-[34] is denoted by $\mathbf{T}^{*}: Y^{*} \rightarrow X^{*}$, which by definition satisfies

$$
\forall x \in X, \forall \phi \in Y^{*}, \quad\langle\mathbf{T} x, \phi\rangle=\left\langle x, \mathbf{T}^{*} \phi\right\rangle
$$

where the notation $\langle y, \phi\rangle$ means the value of the linear functional $\phi$ at $y$. For the given Banach spaces $X$ and $Y$, suppose that there exists unique Banach spaces, denoted by $X_{*}$ and $Y_{*}$, such that their dual spaces $\left(X_{*}\right)^{*}$ and $\left(Y_{*}\right)^{*}$ coincide with $X$ and $Y$, respectively. Then, if there exists an operator $\mathbf{T}_{*}: Y_{*} \rightarrow X_{*}$ such that $\left(\mathbf{T}_{*}\right)^{*}=\mathbf{T}$, then $\mathbf{T}_{*}$ is called the preadjoint [32]-[34] of $\mathbf{T}: X \rightarrow Y$; we can easily see that such an operator $\mathbf{T}_{*}$, if it exists, is unique. It is a fact that $\left\|\mathbf{T}_{*}\right\|=\|\mathbf{T}\|$, where $\left\|\mathbf{T}_{*}\right\|$ denotes the norm of $\mathbf{T}_{*}$ induced from the norms on $Y_{*}$ and $X_{*}$, while $\|\mathbf{T}\|$ denotes the norm of $\mathbf{T}=\left(\mathbf{T}_{*}\right)^{*}$ induced from the (dual) norms on $\left(X_{*}\right)^{*}=X$ and $\left(Y_{*}\right)^{*}=Y$. Not every operator has a preadjoint, but those operators we deal with in this paper do; it suffices to note that for $X=\left(L_{\infty}[0, h)\right)^{\nu}$ and $X=\mathbb{R}_{\infty}^{n}$, a unique $X_{*}$ such that $\left(X_{*}\right)^{*}=X$ is $X_{*}=\left(L_{1}[0, h)\right)^{\nu}$ and $X_{*}=\mathbb{R}_{1}^{n}$, respectively.

Regarding $\left(L_{\infty}[0, h)\right)^{\nu}$, we sometimes drop $\nu$ and slightly abuse a term for simplicity, especially when we refer to the induced norm of an operator; for an operator $\mathbf{T}: X \rightarrow Y$ with $X$ and $Y$ being Banach spaces with norm $\|\cdot\|_{X}$ and $\|\cdot\|_{Y}$, respectively, we call $\|\mathbf{T}\|:=\sup _{x \in X \backslash\{0\}}\|\mathbf{T} x\|_{Y} /\|x\|_{X}$ the $L_{\infty}[0, h)$-induced norm of $\mathbf{T}$ if either $X$ or $Y$ is $\left(L_{\infty}[0, h)\right)^{\nu}$. A similar convention applies when $L_{\infty}[0, h)$ is replaced by a similar space.

The notation $\|\cdot\|$ is used to mean either the $L_{\infty}[0, h)$ norm of a vector function, i.e.,

$$
\|f(\cdot)\|:=\max _{i} \operatorname{ess} \sup _{0 \leq t<h}\left|f_{i}(t)\right|
$$

(or that with $h$ replaced by $h / M$ or $\infty$ ), the $L_{\infty}[0, h)$-induced norm (or that with $h / M$ or $\infty$ instead of $h$ ) of an operator, or the $\infty$-norm of a matrix or a vector, whose distinction will be clear from the context. On the other hand, the notation $\|\cdot\|_{1}$ is used to mean either the $L_{1}[0, h)$ norm of a vector function, i.e.,

$$
\|f(\cdot)\|_{1}:=\sum_{i} \int_{0}^{h}\left|f_{i}(t)\right| d t
$$

(or that with $h$ replaced by $h / M$ or $\infty$ ), the $L_{1}[0, h)$-induced norm (or that with $h / M$ or $\infty$ instead of $h$ ) of an operator, or the 1-norm of a matrix or a vector, whose distinction will also be clear from the context.

For a Banach space $X$, we identify the direct product $\left(X^{m}\right)^{n}$ with $X^{m n}$ when we refer to the norm on the former. We also use the notation $l_{X}$ to denote the space of all $X$ valued sequences, where $X$ is some Banach space. 


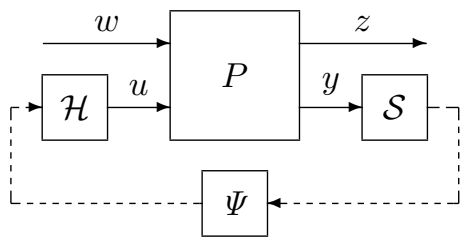

Fig. 1. Sampled-data system $\Sigma_{\mathrm{SD}}$.

\section{III. $L_{1}$ Optimal Control Problem And Lifted REPRESENTATION OF SAMPLED-DATA SYSTEMS}

Let us consider the sampled-data system $\Sigma_{\mathrm{SD}}$ shown in Figure 1, where $P$ denotes the continuous-time generalized plant, while $\Psi, \mathcal{H}$ and $\mathcal{S}$ denote the discrete-time controller, the zero-order hold and the ideal sampler, respectively, operating with sampling period $h$ in a synchronous fashion. Solid lines and dashed lines in Fig. 1 represent continuous-time signals and discrete-time signals, respectively. We suppose that $P$ and $\Psi$ are described respectively by

$$
\begin{gathered}
P:\left\{\begin{array}{l}
\frac{d x}{d t}=A x+B_{1} w+B_{2} u \\
z=C_{1} x+D_{11} w+D_{12} u \\
y=C_{2} x
\end{array}\right. \\
\Psi:\left\{\begin{array}{l}
\psi_{k+1}=A_{\Psi} \psi_{k}+B_{\Psi} y_{k} \\
u_{k}=C_{\Psi} \psi_{k}+D_{\Psi} y_{k}
\end{array}\right.
\end{gathered}
$$

where $x(t) \in \mathbb{R}_{\infty}^{n}, w(t) \in \mathbb{R}_{\infty}^{n_{w}}, u(t) \in \mathbb{R}_{\infty}^{n_{u}}, z(t) \in$ $\mathbb{R}_{\infty}^{n_{z}}, y(t) \in \mathbb{R}_{\infty}^{n_{y}}, \quad \psi_{k} \in \mathbb{R}_{\infty}^{n_{\Psi}}, y_{k}=y(k h)$ and $u(t)=$ $u_{k}(k h \leq t<(k+1) h)$.

This paper studies the so-called $L_{1}$ optimal control problem of the sampled-data system $\Sigma_{\mathrm{SD}}$, i.e., a synthesis method for the stabilizing controller $\Psi$ such that the $L_{\infty}$-induced norm of the mapping ${ }^{2} \mathcal{F}(P, \mathcal{H} \Psi \mathcal{S})$ between $w \in L_{\infty}^{n_{w}}$ and $z \in L_{\infty}^{n_{z}}$ is minimized. Even computing the $L_{\infty}$-induced norm when $P$ and $\Psi$ are given is a difficult problem, to which the idea of fast-sample/fast-hold approximation [29] (or piecewise constant approximation of continuous-time signals) has been applied. Such an approach leads to the associated approximate discretization of $P$ [8]-[10], with which the computation problem is reduced to a discrete-time counterpart (i.e., an $l_{1}$ problem). An important role was played by the lifting technique [21]-[23] in such a direction, through which a synthesis method of $\Psi$ was also developed in [8], [9] with the piecewise constant approximation approach.

Stimulated by the recent success [31] in greatly improving the accuracy in the analysis of the $L_{\infty}$-induced norm via what is called piecewise linear approximation, the present paper aims at developing a theoretical framework by which a more sophisticated synthesis method can be elaborated for the $L_{1}$ optimal control problem of sampled-data systems through a new discretization method of the generalized plant $P$ associated with this extended approximation approach. As it turns out, the new discretization method makes it easier for us to design controllers that successfully reduce the $L_{\infty}$-induced

\footnotetext{
${ }^{2}$ Throughout the paper, $\mathcal{F}(G, H)$ denotes the so-called lower linearfractional-transformation (LFT) given by $G_{11}+G_{12} H\left(I-G_{22} H\right)^{-1} G_{21}$.
}

norm of the closed-loop systems. This could arouse renewed interest for sampled-data $L_{1}$ optimal control for a number of practical applications. The new approach also employs the lifting technique, and thus we begin with its brief review, or the lifted treatment of $\Sigma_{\mathrm{SD}}$.

Given $f(t) \in L_{\infty}^{\nu}$, its lifting $\left\{\widehat{f}_{k}\right\}_{k=0}^{\infty} \in l_{\left(L_{\infty}[0, h)\right)^{\nu}}$ is defined as follows [21]-[23]:

$$
\widehat{f}_{k}(\theta)=f(k h+\theta) \quad(0 \leq \theta<h)
$$

We use $\mathbf{W}_{h}$ to denote lifting: $\left\{\widehat{f}_{k}\right\}_{k=0}^{\infty}=\mathbf{W}_{h} f$. For $\left\{\widehat{f}_{k}\right\}_{k=0}^{\infty} \in$ $l_{\left(L_{\infty}[0, h)\right)^{\nu}}$, we simply call $\left\|\left\{\hat{f}_{k}\right\}\right\|:=\sup _{k}\left\|\widehat{f}_{k}\right\|$ the $l_{[0, h)}^{\infty}$ norm. Since $\mathbf{W}_{h}$ is norm-preserving (i.e., the $l_{[0, h)}^{\infty}$ norm $\left\|\mathbf{W}_{h} f\right\|$ coincides with the $L_{\infty}$ norm $\left.\|f\|\right)$, the induced norm $\|\mathbf{F}\|:=\sup _{x \in X \backslash\{0\}}\|\mathbf{F} x\|_{Y} /\|x\|_{X}$ is sometimes called the $L_{\infty}$-induced norm of $\mathbf{F}$ for simplicity if $\mathbf{F}: X \rightarrow Y$ and

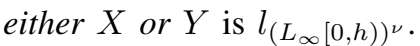

By applying lifting to $w$ and $z$ while discretizing $u$ and $y$, the (partially) lifted representation of the continuous-time generalized plant $P$ is described by

$$
\widehat{P}:\left\{\begin{array}{l}
x_{k+1}=A_{d} x_{k}+\mathbf{B}_{1} \widehat{w}_{k}+B_{2 d} u_{k} \\
\widehat{z}_{k}=\mathbf{C}_{1} x_{k}+\mathbf{D}_{11} \widehat{w}_{k}+\mathbf{D}_{12} u_{k} \\
y_{k}=C_{2 d} x_{k}
\end{array}\right.
$$

with $x_{k}:=x(k h), u_{k}=u(k h)$ and $y_{k}=y(k h)$, the matrices

$$
\begin{aligned}
& A_{d}=\exp (A h): \mathbb{R}_{\infty}^{n} \rightarrow \mathbb{R}_{\infty}^{n} \\
& B_{2 d}=\int_{0}^{h} \exp (A \theta) B_{2} d \theta: \mathbb{R}_{\infty}^{n_{u}} \rightarrow \mathbb{R}_{\infty}^{n} \\
& C_{2 d}=C_{2}: \mathbb{R}_{\infty}^{n} \rightarrow \mathbb{R}_{\infty}^{n_{y}}
\end{aligned}
$$

and the operators

$$
\begin{gathered}
\mathbf{B}_{1} w=\int_{0}^{h} \exp (A(h-\theta)) B_{1} w(\theta) d \theta:\left(L_{\infty}[0, h)\right)^{n_{w}} \rightarrow \mathbb{R}_{\infty}^{n} \\
\begin{aligned}
\left(\mathbf{C}_{1} x\right)(\theta)= & C_{1} \exp (A \theta) x: \mathbb{R}_{\infty}^{n} \rightarrow\left(L_{\infty}[0, h)\right)^{n_{z}} \\
\left(\mathbf{D}_{11} w\right)(\theta) & =\int_{0}^{\theta} C_{1} \exp (A(\theta-\tau)) B_{1} w(\tau) d \tau+D_{11} w(\theta) \\
& :\left(L_{\infty}[0, h)\right)^{n_{w}} \rightarrow\left(L_{\infty}[0, h)\right)^{n_{z}} \\
\left(\mathbf{D}_{12} u_{k}\right)(\theta) & =\int_{0}^{\theta} C_{1} \exp (A(\theta-\tau)) B_{2} d \tau u_{k}+D_{12} u_{k} \\
& : \mathbb{R}_{\infty}^{n_{u}} \rightarrow\left(L_{\infty}[0, h)\right)^{n_{z}}
\end{aligned}
\end{gathered}
$$

Connecting $\Psi$ to the above $\widehat{P}$ leads to the mapping between $\left\{\widehat{w}_{k}\right\}_{k=0}^{\infty}$ and $\left\{\widehat{z}_{k}\right\}_{k=0}^{\infty}$, which we denote by $\mathcal{F}(\widehat{P}, \Psi)$; it coincides with the lifted representation $\mathbf{W}_{h} \mathcal{F}(P, \mathcal{H} \Psi \mathcal{S}) \mathbf{W}_{h}^{-1}$ for the mapping $\mathcal{F}(P, \mathcal{H} \Psi \mathcal{S})$. Since $\mathbf{W}_{h}$ is norm-preserving, we see that the $L_{\infty}$-induced norm $\|\mathcal{F}(P, \mathcal{H} \Psi \mathcal{S})\|$ of $\Sigma_{\mathrm{SD}}$ coincides with the $l_{[0, h)}^{\infty}$-induced norm $\|\mathcal{F}(\widehat{P}, \Psi)\|$.

Let us introduce the notation $\mathbf{M}_{1}:=\left[\begin{array}{ll}\mathbf{C}_{1} & \mathbf{D}_{12}\end{array}\right]$, which can be describe by

$$
\left(\mathbf{M}_{1}\left[\begin{array}{l}
x \\
u
\end{array}\right]\right)(\theta)=C_{0} \exp \left(A_{2} \theta\right)\left[\begin{array}{l}
x \\
u
\end{array}\right]
$$

where

$$
C_{0}:=\left[\begin{array}{ll}
C_{1} & D_{12}
\end{array}\right], \quad A_{2}:=\left[\begin{array}{cc}
A & B_{2} \\
0 & 0
\end{array}\right]
$$


Let us further introduce the (standard lifting-free) discrete-time plant

$$
P_{d}:\left\{\begin{array}{l}
x_{k+1}=A_{d} x_{k}+\eta_{k}+B_{2 d} u_{k} \\
\zeta_{k}=\left[\begin{array}{l}
I \\
0
\end{array}\right] x_{k}+\left[\begin{array}{l}
0 \\
I
\end{array}\right] u_{k} \\
y_{k}=C_{2 d} u_{k}
\end{array}\right.
$$

and denote by $\mathcal{F}\left(P_{d}, \Psi\right)$ the mapping between the discretetime signals $\eta_{k} \in \mathbb{R}_{\infty}^{n}$ and $\zeta_{k} \in \mathbb{R}_{\infty}^{n+n_{u}}$ associated with the closed-loop system obtained by connecting $\Psi$ to the above $P_{d}$. Then, by comparison between this $P_{d}$ and the lifted generalized plant $\widehat{P}$, we see as in [9] that $\mathcal{F}(\widehat{P}, \Psi)$ admits the representation

$$
\mathcal{F}(\widehat{P}, \Psi)=\mathbf{M}_{1} \mathcal{F}\left(P_{d}, \Psi\right) \mathbf{B}_{1}+\mathbf{D}_{11}
$$

Remark 1: In (12), since the left hand side denotes a dynamical system in discrete-time with the lifted input $\left\{\widehat{w}_{k}\right\}_{k=0}^{\infty}$ and output $\left\{\widehat{z}_{k}\right\}_{k=0}^{\infty}$, the operator $\mathbf{B}_{1}$ on the right hand side acts on every $\widehat{w}_{k}$, and $\widehat{z}_{k}$ is associated with the output of $\mathbf{M}_{1}$ for every $k$. Similarly for the interpretation of $\mathbf{D}_{11}$. Similar conventions apply to the following arguments.

Since $\mathcal{F}(\widehat{P}, \Psi)=\mathbf{W}_{h} \mathcal{F}(\widehat{P}, \mathcal{H} \Psi \mathcal{S}) \mathbf{W}_{h}^{-1}$ and $\mathbf{W}_{h}$ is normpreserving as mentioned above, it follows that the $L_{1}$ optimal control problem of $\Sigma_{\mathrm{SD}}$ reduces to the synthesis of $\Psi$ minimizing $\|\mathcal{F}(\widehat{P}, \Psi)\|$. This, however, is still a difficult problem because the operators $\mathbf{B}_{1}$ and $\mathbf{M}_{1}$ (as well as $\mathbf{D}_{11}$ ) cannot be exactly discretized with finite-dimensional matrices in contrast to the $\mathrm{H}_{2}$ and $\mathrm{H}_{\infty}$ problems of sampled-data systems. This paper aims at approximating the operators $\mathbf{B}_{1}, \mathbf{M}_{1}$ and $\mathbf{D}_{11}$ by using the idea of piecewise linear approximation [30], [31], and establishing an associated discretization procedure of the continuous-time generalized plant for the $L_{1}$ optimal control problem of sampled-data systems. More precisely, we provide, by introducing what we call $L_{1}$ discretization of the generalized plant, an (almost) equivalent discrete-time $l_{1}$ optimal control problem together with the associated approximation error analysis.

For the convenience of the reader, brief explanations on the approximation operators used in such arguments are summarized in Table I. Furthermore, the notations used to denote the continuous-time generalized plant and its relevant representations in the derivation of $L_{1}$ discretization are summarized in Table II.

TABLE I

APPROXIMATION OPERATORS USED IN THIS PAPER.

\begin{tabular}{c|cc}
\hline Notation & Meaning & Refer to \\
\hline $\mathbf{J}_{1}^{\prime}$ & Input linearizing operator on $\left[0, h^{\prime}\right)$ & $(17)$ \\
$\mathbf{J}_{M 1} \mathbf{L}_{M}$ & Input piecewise-linearizing operator on $[0, h)$ & $(22)$ \\
\hline $\mathbf{H}_{1}^{\prime}$ & Output linearizing operator on $\left[0, h^{\prime}\right)$ & $(19)$ \\
$\mathbf{L}_{M}^{-1} \mathbf{H}_{M 1}$ & Output piecewise-linearizing operator on $[0, h)$ & $(21)$ \\
\hline $\mathbf{D}_{p 1}^{\prime}$ & Approximate compression operator on $\left[0, h^{\prime}\right)$ & $(20)$ \\
$\mathbf{L}_{M}^{-1} \mathbf{D}_{M 1} \mathbf{L}_{M}$ & Approximate compression operator on $[0, h)$ & $(25)$ \\
\hline
\end{tabular}

\section{Piecewise Linear Approximation of SAMPLED-DATA SYSTEMS}

This section is devoted to providing a method for piecewise linear approximation of sampled-data systems. As a key idea
TABLE II

GENERALIZED PLANT AND ITS RELEVANT REPRESENTATIONS.

\begin{tabular}{c|cc}
\hline Notation & Meaning & Refer to \\
\hline$P$ & Continuous-time generalized plant & $(1)$ \\
$\widehat{P}$ & Lifted representation of $P$ & $(4)$ \\
$\widehat{P}_{M}$ & Fast-lifted representation of $\widehat{P}$ & $(15)$ \\
$\widehat{P}_{M 1}$ & Piecewise-linear approximation of $\widehat{P}$ & $(26)$ \\
$P_{M 1 d}^{[N]}$ & $L_{1}$ discretization of $P$ & $(80)$ \\
\hline
\end{tabular}

in applying the piecewise linear approximation method, we first review fast-lifting [26]. For $M \in \mathbb{N}$ and $h^{\prime}:=h / M$, fast-lifting (with the fast-lifting parameter $M$ ) is defined as the mapping from $f \in\left(L_{\infty}[0, h)\right)^{\nu}$ (or $f \in\left(L_{1}[0, h)\right)^{\nu}$ )

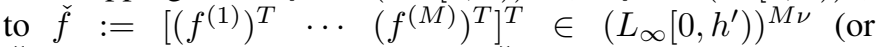
$\check{f} \in\left(L_{1}\left[0, h^{\prime}\right)\right)^{M \nu}$ ), denoted by $\check{f}=\mathbf{L}_{M} f$ (irrespective of the underlying space for $f$, for notational simplicity), where

$$
f^{(i)}\left(\theta^{\prime}\right):=f\left((i-1) h^{\prime}+\theta^{\prime}\right) \quad\left(0 \leq \theta^{\prime}<h^{\prime}\right)
$$

It is easy to see that $\mathbf{L}_{M}$ is norm-preserving. It readily follows from this property that

$$
\|\mathcal{F}(\widehat{P}, \Psi)\|=\left\|\mathbf{L}_{M} \mathcal{F}(\widehat{P}, \Psi) \mathbf{L}_{M}^{-1}\right\|
$$

where the right-hand side means the $l_{\left[0, h^{\prime}\right)}^{\infty}$-induced norm. Let us consider applying fast-lifting on $\widehat{w}_{k}$ and $\widehat{z}_{k}$ in the (partially) lifted generalized plant $\widehat{P}$, and consider its fastlifted counterpart

$$
\widehat{P}_{M}=\operatorname{diag}\left[\mathbf{L}_{M}, I\right] \widehat{P} \operatorname{diag}\left[\mathbf{L}_{M}^{-1}, I\right]
$$

Then, we see that $\mathbf{L}_{M} \mathcal{F}(\widehat{P}, \Psi) \mathbf{L}_{M}^{-1}=\mathcal{F}\left(\widehat{P}_{M}, \Psi\right)$, and it admits the representation

$$
\mathcal{F}\left(\widehat{P}_{M}, \Psi\right)=\mathbf{L}_{M} \mathbf{M}_{1} \mathcal{F}\left(P_{d}, \Psi\right) \mathbf{B}_{1} \mathbf{L}_{M}^{-1}+\mathbf{L}_{M} \mathbf{D}_{11} \mathbf{L}_{M}^{-1}
$$

which we call the fast-lifted representation of $\Sigma_{\mathrm{SD}}$.

We are in a position to review the piecewise linear approximation treatment of the operators $\mathbf{B}_{1}, \mathbf{M}_{1}$ and $\mathbf{D}_{11}$ in the above fast-lifted representation (16), which immediately leads us to piecewise linear approximation of $\Sigma_{\mathrm{SD}}$. Such treatment was developed in [30], [31] for analysis problems, but it was far from straightforward to extend the method in [30] in such a way that the sampled-data $L_{1}$ controller design problem can be dealt with. The present paper employs essentially the same approximation of these operators as a fundamental tool but aims at taking a completely new approach so that we can establish a discretization procedure of the continuous-time generalized plant for the sampled-data $L_{1}$ control problem. As in existing studies, this is a problem that aims at minimizing the $L_{\infty}$-induced norm of $\Sigma_{\mathrm{SD}}$, for which our discretization of the generalized plant, together with the associated approximation error analysis, provides an approach that reduces the problem to an (almost) equivalent discretetime $l_{1}$ control problem (which is the problem of minimizing the $l_{\infty}$-induced norm).

To describe the approximation treatment, we first introduce with $h^{\prime}=h / M$ the 'linearizing' operator $\mathbf{J}_{1}^{\prime}$ : $\left(L_{\infty}\left[0, h^{\prime}\right)\right)^{n_{w}} \rightarrow\left(L_{\infty}\left[0, h^{\prime}\right)\right)^{n_{w}}$ [30], [31] (by which we 
mean that $\mathbf{J}_{1}^{\prime} w$ is always a linear function) described by

$$
\left(\mathbf{J}_{1}^{\prime} w\right)\left(\theta^{\prime}\right)=\int_{0}^{h^{\prime}} f_{0}\left(\tau^{\prime}\right) w\left(\tau^{\prime}\right) d \tau^{\prime}+\theta^{\prime} \int_{0}^{h^{\prime}} f_{1}\left(\tau^{\prime}\right) w\left(\tau^{\prime}\right) d \tau^{\prime}
$$

with the scalar-valued functions

$$
f_{0}\left(\tau^{\prime}\right)=-\frac{6}{\left(h^{\prime}\right)^{2}} \tau^{\prime}+\frac{4}{h^{\prime}}, f_{1}\left(\tau^{\prime}\right)=\frac{12}{\left(h^{\prime}\right)^{3}} \tau^{\prime}-\frac{6}{\left(h^{\prime}\right)^{2}}
$$

$\mathbf{J}_{1}^{\prime}$ is used to approximate the input of $\mathbf{B}_{1}$ with a piecewise linear function and is tailored to possess important properties in terms of some Taylor expansion arguments [31], in addition to the property that $\mathbf{J}_{1}^{\prime} w=w$ for any linear function $w$; such properties will be used in the proof of our main results. See [31] for more details. We further introduce another 'linearizing' operator $\mathbf{H}_{1}^{\prime}:\left(L_{\infty}\left[0, h^{\prime}\right)\right)^{n_{z}} \rightarrow$ $\left(L_{\infty}\left[0, h^{\prime}\right)\right)^{n_{z}}$, as well as the operator $\mathbf{D}_{p 1}^{\prime}:\left(L_{\infty}\left[0, h^{\prime}\right)\right)^{n_{w}} \rightarrow$ $\left(L_{\infty}\left[0, h^{\prime}\right)\right)^{n_{z}}$ [30], [31] (although the introduction of $\mathbf{H}_{1}^{\prime}$ was only implicit there), described by

$$
\begin{aligned}
& \left(\mathbf{H}_{1}^{\prime} z\right)\left(\theta^{\prime}\right)=z(0)+\left.\theta^{\prime} \frac{d z\left(\tau^{\prime}\right)}{d \tau^{\prime}}\right|_{\tau^{\prime}=0}\left(0 \leq \theta^{\prime}<h^{\prime}\right) \\
& \left(\mathbf{D}_{p 1}^{\prime} w\right)\left(\theta^{\prime}\right)=\int_{0}^{\theta^{\prime}} C_{1} B_{1} w\left(\tau^{\prime}\right) d \tau^{\prime}+D_{11} w\left(\theta^{\prime}\right)\left(0 \leq \theta^{\prime}<h^{\prime}\right)
\end{aligned}
$$

Obviously, $\mathbf{H}_{1}^{\prime}$ could be interpreted as an operator producing a linear function that preserves the value and derivative of the input at $\theta^{\prime}=0$, and thus $\mathbf{H}_{1}^{\prime} z=z$ for any linear function $z$ on $\left[0, h^{\prime}\right)$. Strictly speaking, $\mathbf{H}_{1}^{\prime}$ is not an operator on $\left(L_{\infty}\left[0, h^{\prime}\right)\right)^{n_{z}}$ but on its subspace of functions continuous and (right) differentiable at time 0 . However, this issue causes no problems since $\mathbf{H}_{1}^{\prime}$ is used for approximating $\mathbf{M}_{1}$ (or its output with a piecewise linear function) and operates only on its output. In contrast, approximation of $\mathbf{B}_{1}$ should take into account that its input may be discontinuous, and this is why the other more involved linearizing operator $\mathbf{J}_{1}^{\prime}$ given above is used. $\mathbf{D}_{p 1}^{\prime}$ is used for approximating $\mathbf{D}_{11}$ as in [30], [31], and it corresponds to applying constant approximation to the kernel function associated with the compact portion of the compression operator defined on $\left[0, h^{\prime}\right)$. The details of our approximation treatment employing these operators is as follows.

Following the basic ideas mentioned above, we consider replacing $\mathbf{L}_{M} \mathbf{M}_{1}$ and $\mathbf{B}_{1} \mathbf{L}_{M}^{-1}$ in (16) with $\overline{\mathbf{H}_{1}^{\prime}} \mathbf{L}_{M} \mathbf{M}_{1}$ and $\mathbf{B}_{1} \mathbf{L}_{M}^{-1} \overline{\mathbf{J}_{1}^{\prime}}$, respectively, (i.e., $\mathbf{B}_{1}$ and $\mathbf{M}_{1}$ are approximated by $\mathbf{B}_{1} \mathbf{L}_{M}^{-1} \overline{\mathbf{J}_{1}^{\prime}} \mathbf{L}_{M}$ and $\mathbf{L}_{M}^{-1} \overline{\mathbf{H}_{1}^{\prime}} \mathbf{L}_{M} \mathbf{M}_{1}$, respectively) where $\overline{(\cdot)}$ denotes $\operatorname{diag}[(\cdot), \cdots,(\cdot)]$ consisting of $M$ copies of $(\cdot)$. To facilitate such treatment, let us introduce the operators $\mathbf{H}_{M 1}$ and $\mathbf{J}_{M 1}$ by

$$
\begin{aligned}
& \mathbf{H}_{M 1}=\overline{\mathbf{H}_{1}^{\prime}} \mathbf{L}_{M}:\left(L_{\infty}[0, h)\right)^{n_{z}} \rightarrow\left(L_{\infty}\left[0, h^{\prime}\right)\right)^{M n_{z}} \\
& \mathbf{J}_{M 1}=\mathbf{L}_{M}^{-1} \overline{\mathbf{J}_{1}^{\prime}}:\left(L_{\infty}\left[0, h^{\prime}\right)\right)^{M n_{w}} \rightarrow\left(L_{\infty}[0, h)\right)^{n_{w}}
\end{aligned}
$$

Remark 2: With $\mathbf{B}_{1}^{\prime}$ introduced shortly, $\mathbf{B}_{1} \mathbf{L}_{M}^{-1}$ can be described as $A_{d M}^{\prime} \overline{\mathbf{B}_{1}^{\prime}}$ with an appropriate matrix $A_{d M}^{\prime}$. Hence, approximating $\mathbf{B}_{1} \mathbf{L}_{M}^{-1}$ with $\mathbf{B}_{1} \mathbf{L}_{M}^{-1} \overline{\mathbf{J}_{1}^{\prime}}$ is equivalent to approximating $\mathbf{B}_{1}^{\prime}$ with $\mathbf{B}_{1}^{\prime} \mathbf{J}_{1}^{\prime}$ (i.e., approximating the input of $\mathbf{B}_{1}^{\prime}$ with a linear function). Similarly, approximating $\mathbf{L}_{M} \mathbf{M}_{1}$ with
$\overline{\mathbf{H}_{1}^{\prime}} \mathbf{L}_{M} \mathbf{M}_{1}$ is equivalent to approximating $\mathbf{M}_{1}^{\prime}$ with $\mathbf{H}_{1}^{\prime} \mathbf{M}_{1}^{\prime}$ (i.e., approximating the output of $\mathbf{M}_{1}^{\prime}$ with a linear function), where $\mathbf{M}_{1}^{\prime}$ will also be given below.

Next, to facilitate the treatment of $\mathbf{L}_{M} \mathbf{D}_{11} \mathbf{L}_{M}^{-1}$ in (16), we introduce the operators $\mathbf{B}_{1}^{\prime}, \mathbf{M}_{1}^{\prime}$ and $\mathbf{D}_{11}^{\prime}$ defined as $\mathbf{B}_{1}, \mathbf{M}_{1}$ and $\mathbf{D}_{11}$ respectively, with the horizon $[0, h)$ replaced by $\left[0, h^{\prime}\right)$, as well as the matrices

$$
\begin{aligned}
& A_{2 d}^{\prime}=\exp \left(A_{2} h^{\prime}\right): \mathbb{R}_{\infty}^{n+n_{u}} \rightarrow \mathbb{R}_{\infty}^{n+n_{u}} \\
& J=\left[\begin{array}{l}
I \\
0
\end{array}\right]: \mathbb{R}_{\infty}^{n+n_{u}} \rightarrow \mathbb{R}_{\infty}^{n}
\end{aligned}
$$

Then, it is easy to see that $\mathbf{L}_{M} \mathbf{D}_{11} \mathbf{L}_{M}^{-1}$ is described by

$$
\mathbf{L}_{M} \mathbf{D}_{11} \mathbf{L}_{M}^{-1}=\overline{\mathbf{M}_{1}^{\prime}} \Delta_{M}^{0} \overline{\mathbf{B}_{1}^{\prime}}+\overline{\mathbf{D}_{11}^{\prime}}
$$

(see, e.g., [26]), where

$$
\Delta_{M}^{0}:=\left[\begin{array}{cccc}
0 & 0 & \cdots & 0 \\
J & \ddots & \ddots & \vdots \\
\vdots & \ddots & \ddots & 0 \\
\left(A_{2 d}^{\prime}\right)^{M-2} J & \cdots & J & 0
\end{array}\right]
$$

Applying once again the aforementioned ideas to (23), we further define the operator

$$
\begin{aligned}
\mathbf{D}_{M 1}= & \overline{\mathbf{H}_{1}^{\prime}} \overline{\mathbf{M}_{1}^{\prime}} \Delta_{M}^{0} \overline{\mathbf{B}_{1}^{\prime}} \overline{\mathbf{J}_{1}^{\prime}}+\overline{\mathbf{D}_{p 1}^{\prime}}: \\
& \left(L_{\infty}\left[0, h^{\prime}\right)\right)^{M n_{w}} \rightarrow\left(L_{\infty}\left[0, h^{\prime}\right)\right)^{M n_{z}}
\end{aligned}
$$

What has been done up to now is that the input and output of $\mathbf{M}_{1} \mathcal{F}\left(P_{d}, \Psi\right) \mathbf{B}_{1}$ in (16) are approximated by piecewise linear functions, similar treatment has been done on the first term on the right hand side of (23), and the second term of (23) was approximated by $\overline{\mathbf{D}_{p 1}^{\prime}}$. The last treatment has followed a similar technique in [30], [31]. To summarize, we have introduced the following approximation of $\mathcal{F}\left(\widehat{P}_{M}, \Psi\right)$ :

$$
\mathcal{F}\left(\widehat{P}_{M 1}, \Psi\right):=\mathbf{H}_{M 1} \mathbf{M}_{1} \mathcal{F}\left(P_{d}, \Psi\right) \mathbf{B}_{1} \mathbf{J}_{M 1}+\mathbf{D}_{M 1}
$$

We call it piecewise linear approximation of the sampleddata system $\Sigma_{\mathrm{SD}}$, which alleviates the difficulty in computing $\|\mathcal{F}(\widehat{P}, \Psi)\|=\left\|\mathcal{F}\left(\widehat{P}_{M}, \Psi\right)\right\|$.

\section{Error Analysis of Piecewise Linear APPROXIMATION}

This section is devoted to showing that the error in piecewise linear approximation converges to 0 at the rate of $1 / M^{2}$ as $M \rightarrow \infty$. To evaluate the error in the approximation of $\|\mathcal{F}(\widehat{P}, \Psi)\|=\left\|\mathcal{F}\left(\widehat{P}_{M}, \Psi\right)\right\|$ by $\left\|\mathcal{F}\left(\widehat{P}_{M 1}, \Psi\right)\right\|$, we first introduce 'finite-rank portions' of $\mathcal{F}\left(\widehat{P}_{M}, \Psi\right)$ in (16) and $\mathcal{F}\left(\widehat{P}_{M 1}, \Psi\right)$ in (26) given respectively by

$$
\begin{aligned}
\mathcal{F}^{0}\left(\widehat{P}_{M}, \Psi\right) & :=\mathbf{L}_{M} \mathbf{M}_{1} \mathcal{F}\left(P_{d}, \Psi\right) \mathbf{B}_{1} \mathbf{L}_{M}^{-1} \\
& =\mathcal{F}\left(\widehat{P}_{M}, \Psi\right)-\mathbf{L}_{M} \mathbf{D}_{11} \mathbf{L}_{M}^{-1} \\
\mathcal{F}^{0}\left(\widehat{P}_{M 1}, \Psi\right) & :=\mathbf{H}_{M 1} \mathbf{M}_{1} \mathcal{F}\left(P_{d}, \Psi\right) \mathbf{B}_{1} \mathbf{J}_{M 1} \\
& =\mathcal{F}\left(\widehat{P}_{M 1}, \Psi\right)-\mathbf{D}_{M 1}
\end{aligned}
$$

Comparing the above equations, we see that evaluating $\mathbf{J}_{M 1}-$ $\mathbf{L}_{M}^{-1}$ and $\mathbf{H}_{M 1}-\mathbf{L}_{M}$ is a key in the error analysis. The 
following lemma is relevant to such evaluation and plays a key role in our discussions.

Lemma 1: Suppose that $\left(A, B_{1}\right)$ is controllable and $\left(C_{0}, A_{2}\right)$ is observable. Then, we have the following properties regarding the preadjoints $\mathbf{J}_{M 1 *}$ and $\mathbf{B}_{1 *}$ and the operators $\mathbf{H}_{M 1}$ and $\mathbf{M}_{1}$.

a) There exists a constant $K_{B}$ such that

$$
\left\|\left.\left(\mathbf{L}_{M}-\mathbf{J}_{M 1 *}\right)\right|_{R\left(\mathbf{B}_{1 *}\right)}\right\|_{1} \leq \frac{K_{B}}{M^{2}}
$$

where $R\left(\mathbf{B}_{1 *}\right)$ is viewed as a subset of $\left(L_{1}[0, h)\right)^{n_{w}}$.

b) There exists a constant $K_{C}$ such that

$$
\left\|\left.\left(\mathbf{L}_{M}-\mathbf{H}_{M 1}\right)\right|_{R\left(\mathbf{M}_{1}\right)}\right\| \leq \frac{K_{C}}{M^{2}}
$$

where $R\left(\mathbf{M}_{1}\right)$ is viewed as a subset of $\left(L_{\infty}[0, h)\right)^{n_{z}}$.

Remark 3: The two norms $\|\cdot\|_{1}$ and $\|\cdot\|$ in Lemma 1 mean the $L_{1}\left[0, h^{\prime}\right)$-induced norm and the $L_{\infty}\left[0, h^{\prime}\right)$-induced norm, respectively. From the definition of the preadjoint in Section II, $\mathbf{J}_{M 1 *}:\left(L_{1}[0, h)\right)^{n_{w}} \rightarrow\left(L_{1}\left[0, h^{\prime}\right)\right)^{M n_{w}}$ is given by

$$
\mathbf{J}_{M 1 *}:=\overline{\mathbf{J}_{1 *}^{\prime}} \mathbf{L}_{M}
$$

where the preadjoint $\mathbf{J}_{1 *}^{\prime}$ is given by

$$
\left(\mathbf{J}_{1 *}^{\prime} w\right)\left(\theta^{\prime}\right)=f_{0}\left(\theta^{\prime}\right) \int_{0}^{h^{\prime}} w\left(\tau^{\prime}\right) d \tau^{\prime}+f_{1}\left(\theta^{\prime}\right) \int_{0}^{h^{\prime}} \tau^{\prime} w\left(\tau^{\prime}\right) d \tau^{\prime}
$$

Remark 4: If we note (31), it is not hard to see that the claim (29) can be roughly restated as the assertion that $\left\|\left(I-\overline{\mathbf{J}_{1 *}^{\prime}}\right) \mathbf{L}_{M} \mathbf{B}_{1 *}\right\|_{1}=\left\|\mathbf{B}_{1} \mathbf{L}_{M}^{-1}\left(I-\overline{\mathbf{J}_{1}^{\prime}}\right)\right\|$ can be made arbitrarily small with the order $1 / M^{2}$ as $M$ tends to $\infty$. Roughly speaking, the latter assertion could be interpreted as a result of the following observation: if $M$ is large enough, the input of $\mathbf{B}_{1}$ could be approximated by a piecewise linear function with $M$ segments, causing only slight effects on its output, because of the 'low pass' nature of the integral operator $\mathbf{B}_{1}$. The claim (30) also has a similar interpretation. Regarding rigorous proof of Lemma 1, we mostly follow similar arguments to the proof of Lemma 4 in [9], which is a result concerned with the FSFH (or piecewise constant) approximation technique [29]. In [9], however, integral inequalities are used to establish the associated convergence rate (see (18), (19) and (20) in [9] for details). Since these integral inequalities cannot be used for establishing (29) and (30), the proof of Lemma 1 uses instead a Taylor expansion technique in a similar way to [31]. The details are given in Appendix A.

Remark 5: The controllability and observability assumptions in Lemma 1 are only for the ease in the proof, and can in fact be removed. This is because we can always replace these pairs with controllable and observable ones, without changing the ranges $R\left(\mathbf{B}_{1 *}\right)$ and $R\left(\mathbf{M}_{1}\right)$.

We have the following key result from Lemma 1.

Proposition 1: There exists a constant $K_{0}$ independent of $\Psi$, such that

$$
\left\|\mathcal{F}^{0}\left(\widehat{P}_{M 1}, \Psi\right)-\mathcal{F}^{0}\left(\widehat{P}_{M}, \Psi\right)\right\| \leq \frac{K_{0}}{M^{2}}\left\|\mathcal{F}^{0}\left(\widehat{P}_{M}, \Psi\right)\right\|
$$

Remark 6: We could also establish a similar inequality with $\mathcal{F}^{0}\left(\widehat{P}_{M}, \Psi\right)$ replaced by $\mathcal{F}^{0}\left(\widehat{P}_{M 1}, \Psi\right)$ on the right hand side, if we use slightly modified versions of inequalities (29) and (30). However, the proof of the modified inequalities becomes more involved as in [9], which indeed derived such versions of inequalities corresponding to the FSFH approximation (or piecewise constant approximation). We stress that our following arguments successfully utilize the simpler inequality (33) and circumvent such involved arguments.

Proof: We first deal with the approximation on the output side. Let us introduce the following 'finite-rank portion' of $\mathcal{F}(\widehat{P}, \Psi)$ :

$$
\mathcal{F}^{0}(\widehat{P}, \Psi):=\mathbf{M}_{1} \mathcal{F}\left(P_{d}, \Psi\right) \mathbf{B}_{1}
$$

From the second assertion of Lemma 1, we have

$$
\begin{aligned}
& \left\|\mathcal{F}^{0}\left(\widehat{P}_{M}, \Psi\right)-\mathbf{H}_{M 1} \mathcal{F}^{0}(\widehat{P}, \Psi) \mathbf{L}_{M}^{-1}\right\| \\
= & \left\|\mathbf{L}_{M} \mathbf{M}_{1} \mathcal{F}\left(P_{d}, \Psi\right) \mathbf{B}_{1} \mathbf{L}_{M}^{-1}-\mathbf{H}_{M 1} \mathbf{M}_{1} \mathcal{F}\left(P_{d}, \Psi\right) \mathbf{B}_{1} \mathbf{L}_{M}^{-1}\right\| \\
= & \left\|\left.\left(\mathbf{L}_{M}-\mathbf{H}_{M 1}\right)\right|_{R\left(\mathbf{M}_{1}\right)} \mathbf{M}_{1} \mathcal{F}\left(P_{d}, \Psi\right) \mathbf{B}_{1} \mathbf{L}_{M}^{-1}\right\| \\
\leq & \frac{K_{C}}{M^{2}}\left\|\mathbf{M}_{1} \mathcal{F}\left(P_{d}, \Psi\right) \mathbf{B}_{1} \mathbf{L}_{M}^{-1}\right\| \\
= & \frac{K_{C}}{M^{2}}\left\|\mathcal{F}^{0}\left(\widehat{P}_{M}, \Psi\right)\right\|
\end{aligned}
$$

In particular, this implies

$$
\left\|\mathbf{H}_{M 1} \mathcal{F}^{0}(\widehat{P}, \Psi) \mathbf{L}_{M}^{-1}\right\| \leq\left(1+\frac{K_{C}}{M^{2}}\right)\left\|\mathcal{F}^{0}\left(\widehat{P}_{M}, \Psi\right)\right\|
$$

We next deal with the approximation on the input side. It follows from the first assertion of Lemma 1 that

$$
\begin{aligned}
& \left\|\mathbf{H}_{M 1} \mathcal{F}^{0}(\widehat{P}, \Psi) \mathbf{L}_{M}^{-1}-\mathcal{F}^{0}\left(\widehat{P}_{M 1}, \Psi\right)\right\| \\
= & \left\|\mathbf{H}_{M 1} \mathbf{M}_{1} \mathcal{F}\left(P_{d}, \Psi\right) \mathbf{B}_{1} \mathbf{L}_{M}^{-1}-\mathbf{H}_{M 1} \mathbf{M}_{1} \mathcal{F}\left(P_{d}, \Psi\right) \mathbf{B}_{1} \mathbf{J}_{M 1}\right\| \\
= & \left\|\left(\mathbf{H}_{M 1} \mathbf{M}_{1} \mathcal{F}\left(P_{d}, \Psi\right)\right) \mathbf{B}_{1}\left(\mathbf{L}_{M}^{-1}-\mathbf{J}_{M 1}\right)\right\| \\
= & \left\|\left(\mathbf{L}_{M}-\mathbf{J}_{M 1 *}\right) \mathbf{B}_{1 *}\left(\mathbf{H}_{M 1} \mathbf{M}_{1} \mathcal{F}\left(P_{d}, \Psi\right)\right)_{*}\right\|_{1} \\
= & \left\|\left.\left(\mathbf{L}_{M}-\mathbf{J}_{M 1 *}\right)\right|_{R\left(\mathbf{B}_{1 *}\right)}\left(\mathbf{H}_{M 1} \mathbf{M}_{1} \mathcal{F}\left(P_{d}, \Psi\right) \mathbf{B}_{1}\right)_{*}\right\|_{1} \\
\leq & \frac{K_{B}}{M^{2}}\left\|\mathbf{H}_{M 1} \mathbf{M}_{1} \mathcal{F}\left(P_{d}, \Psi\right) \mathbf{B}_{1}\right\| \\
= & \frac{K_{B}}{M^{2}}\left\|\mathbf{H}_{M 1} \mathcal{F}^{0}(\widehat{P}, \Psi) \mathbf{L}_{M}^{-1}\right\|
\end{aligned}
$$

Combining (35), (37) and (36) leads to (33) with $K_{0}:=$ $K_{B}\left(1+K_{C}\right)+K_{C}$. This completes the proof.

In view of (27) and (28), it is also helpful to evaluate $\mathbf{D}_{M 1}-$ $\mathbf{L}_{M} \mathbf{D}_{11} \mathbf{L}_{M}^{-1}$, for which we have the following result.

Lemma 2 ( [30], [31]): The inequality

$$
\left\|\mathbf{D}_{M 1}-\mathbf{L}_{M} \mathbf{D}_{11} \mathbf{L}_{M}^{-1}\right\| \leq \frac{K_{1}}{M^{2}}
$$

holds with $K_{1}$ defined as

$$
\begin{aligned}
K_{1}:=\frac{1}{2}\left\|C_{1}\right\| \cdot\|A\|^{2} \cdot & \left\|B_{1}\right\| e^{2\|A\| h} h^{3}\left(1+e^{\|A\| h}+\|A\| h\right) \\
& +\frac{1}{2}\left\|C_{1}\right\| \cdot\|A\| \cdot\left\|B_{1}\right\| h^{2} e^{\|A\| h}
\end{aligned}
$$

We are in a position to give the following main result on the error analysis of piecewise linear approximation.

Theorem 2: The following inequality holds:

$$
\begin{aligned}
\left(1-\frac{K_{0}}{M^{2}}\right)\|\mathcal{F}(\widehat{P}, \Psi)\|-\frac{K_{1}}{M^{2}} \leq\left\|\mathcal{F}\left(\widehat{P}_{M 1}, \Psi\right)\right\| \\
\leq\left(1+\frac{K_{0}}{M^{2}}\right)\|\mathcal{F}(\widehat{P}, \Psi)\|+\frac{K_{1}}{M^{2}}
\end{aligned}
$$


Proof: A key in the proof is to show that $\left\|\mathcal{F}^{0}(\widehat{P}, \Psi)\right\| \leq$ $\|\mathcal{F}(\widehat{P}, \Psi)\|$. This inequality follows from the properties of $L_{\infty}[0, h)$ if we note that the infinite (Toeplitz) matrix representation of the input/output relation of $\mathcal{F}^{0}(\widehat{P}, \Psi)=$ $\mathbf{M}_{1} \mathcal{F}\left(P_{d}, \Psi\right) \mathbf{B}_{1}$ is strictly block lower triangular (with respect to the partitioning associated with $\widehat{w}_{k}$ and $\widehat{z}_{k}$ ) because of the structure of $P_{d}$ (note that (11) has no direct feedthrough matrix between $\eta_{k}$ and $\zeta_{k}$ ); this infinite matrix obviously has no overlap of nonzero entries with the infinite matrix representation of $\mathcal{F}(\widehat{P}, \Psi)-\mathcal{F}^{0}(\widehat{P}, \Psi)=\mathbf{D}_{11}$ (which is nothing but the infinite block diagonal matrix with all diagonal entries given by $\mathbf{D}_{11}$ ).

It follows form Proposition 1 and Lemma 2 that

$$
\begin{aligned}
& \left\|\mathcal{F}\left(\widehat{P}_{M 1}, \Psi\right)-\mathcal{F}\left(\widehat{P}_{M}, \Psi\right)\right\| \\
\leq & \frac{K_{0}}{M^{2}}\left\|\mathcal{F}^{0}\left(\widehat{P}_{M}, \Psi\right)\right\|+\frac{K_{1}}{M^{2}} \\
\leq & \frac{K_{0}}{M^{2}}\left\|\mathcal{F}\left(\widehat{P}_{M}, \Psi\right)\right\|+\frac{K_{1}}{M^{2}}
\end{aligned}
$$

Since $\left\|\mathcal{F}\left(\widehat{P}_{M}, \Psi\right)\right\|=\|\mathcal{F}(\widehat{P}, \Psi)\|$, the assertion follows immediately.

Theorem 2 clearly implies that the approximation error in the piecewise linear approximation converges to 0 at the rate of $1 / M^{2}$ as $M \rightarrow \infty$, when the $L_{\infty}$-induced norm of $\Sigma_{\mathrm{SD}}$ is computed for a fixed controller $\Psi$. Theorem 2 also gives a theoretical basis for such an indirect and approximate approach to $L_{1}$-optimal controller synthesis for $\Sigma_{\mathrm{SD}}$ that seeks for $\Psi$ minimizing $\left\|\mathcal{F}\left(\widehat{P}_{M 1}, \Psi\right)\right\|$ for a sufficiently large $M$. To see this, let

$$
\gamma_{\text {opt }}:=\inf _{\Psi}\|\mathcal{F}(\widehat{P}, \Psi)\|
$$

and take an $M$. Suppose that $\Psi_{M}$ is an $\varepsilon$-suboptimal controller with respect to $\left\|\mathcal{F}\left(\widehat{P}_{M 1}, \Psi\right)\right\|$, i.e., $\left\|\mathcal{F}\left(\widehat{P}_{M 1}, \Psi_{M}\right)\right\| \leq \gamma_{M}+$ $\epsilon(\epsilon>0)$, where $\gamma_{M}:=\inf _{\Psi}\left\|\mathcal{F}\left(\widehat{P}_{M 1}, \Psi\right)\right\|$. Let $M_{0} \in \mathbb{N}$ be the minimum such that $M_{0}^{2}>K_{0}$. Then, for $M \geq M_{0}$, the first inequality of (40) implies that

$$
\begin{aligned}
\gamma_{\mathrm{opt}} & \leq\left\|\mathcal{F}\left(\widehat{P}, \Psi_{M}\right)\right\| \\
& \leq\left(1+\frac{K_{0}}{M^{2}-K_{0}}\right)\left\|\mathcal{F}\left(\widehat{P}_{M 1}, \Psi_{M}\right)\right\|+\frac{K_{1}}{M^{2}-K_{0}} \\
& \leq\left(1+\frac{K_{0}}{M^{2}-K_{0}}\right)\left(\gamma_{M}+\epsilon\right)+\frac{K_{1}}{M^{2}-K_{0}}
\end{aligned}
$$

On the other hand, it follows from the second inequality of (40) that

$$
\gamma_{M} \leq\left(1+\frac{K_{0}}{M^{2}}\right) \gamma_{\mathrm{opt}}+\frac{K_{1}}{M^{2}}
$$

Substituting this into (43) and taking a sufficiently large $M$ such that $M \geq M_{0}$, we see that

$$
\gamma_{\mathrm{opt}} \leq\left\|\mathcal{F}\left(\widehat{P}, \Psi_{M}\right)\right\| \leq \gamma_{\mathrm{opt}}+\epsilon+\frac{X}{M^{2}}
$$

where

$$
X:=\frac{2 K_{0} \gamma_{\mathrm{opt}}}{1-K_{0} / M_{0}^{2}}+\frac{2 K_{1}}{1-K_{0} / M_{0}^{2}}+\frac{K_{0} \epsilon}{1-K_{0} / M_{0}^{2}}
$$

Since $\epsilon>0$ is arbitrary, taking a suboptimal $\Psi_{M}$ with respect to $\left\|\mathcal{F}\left(\widehat{P}_{M 1}, \Psi\right)\right\|$ (sufficiently close to the infimal performance) and letting $M$ sufficiently large is ensured to lead to a method for $L_{1}$-optimal controller synthesis for $\Sigma_{\mathrm{SD}}$. By (45), we could say that the convergence of $\Psi_{M}$ is in the order of $1 / M^{2}$. We will further show in Section VI that the synthesis problem of a suboptimal $\Psi_{M}$ can be (almost equivalently) reduced to a discrete-time $l_{1}$ optimal control problem.

Remark 7: Because of the slight difference stated in Remark 6, our inequality (40) may not appear to be a completely direct extension of a similar inequality in [9]. However, the inequality in [9] is also aimed at showing the convergence rate, and it was shown to be at the rate of $1 / M$ for the FSFH (or piecewise constant) approximation. Since our inequality shows that the convergence rate is $1 / M^{2}$, we see that our piecewise linear approximation is much better.

\section{Discretization of Piecewise Linear APPROXIMATION OF THE SAMPLED-DATA SySTEM $\Sigma_{\mathrm{SD}}$}

The preceding section showed a promising aspect of dealing with the piecewise linear approximation $\mathcal{F}\left(\widehat{P}_{M 1}, \Psi\right)$. To exploit this approximation, however, we obviously need to have an explicit method for computing $\left\|\mathcal{F}\left(\widehat{P}_{M 1}, \Psi\right)\right\|$, and this section is devoted to giving such a method. More precisely, we show that a discretized generalized plant (for the continuoustime generalized plant $P$ ) can be introduced that is useful for computing $\left\|\mathcal{F}\left(\widehat{P}_{M 1}, \Psi\right)\right\|$. We further show that the discretized generalized plant together with associated error analysis converts the synthesis problem of an $L_{1}$-optimal controller $\Psi$ for the sampled-data system $\Sigma_{\mathrm{SD}}$ into the discrete-time synthesis problem of an $l_{1}$-optimal controller.

To derive such a discretized generalized plant, we recall that $\mathcal{F}\left(\widehat{P}_{M 1}, \Psi\right)$ is given by (26) and consider replacing $\mathbf{B}_{1} \mathbf{J}_{M 1}, \mathbf{H}_{M 1} \mathbf{M}_{1}$ and $\mathbf{D}_{M 1}$ with finite-dimensional matrices.

\section{A. Approximation of the unit ball image of $\mathbf{B}_{1} \mathbf{J}_{M 1}$}

This subsection provides a method for approximating the closed unit ball image of $\mathbf{B}_{1} \mathbf{J}_{M 1}$ with that of a matrix in the $l_{\infty}$ sense. To this end, we first review the operation of $\mathbf{B}_{1} \mathbf{J}_{M 1}=\mathbf{B}_{1} \mathbf{L}_{M}^{-1} \overline{\mathbf{J}_{1}^{\prime}}$. For $w=\left[\left(w^{(1)}\right)^{T}, \cdots,\left(w^{(M)}\right)^{T}\right]^{T} \in$ $\left(L_{\infty}\left[0, h^{\prime}\right)\right)^{M n_{w}}$, we have

$$
\begin{aligned}
\mathbf{B}_{1} \mathbf{J}_{M 1} w & =\left[\begin{array}{lll}
\left(A_{d}^{\prime}\right)^{M-1} & \ldots & I
\end{array}\right] \overline{\mathbf{B}_{1}^{\prime}} \overline{\mathbf{J}_{1}^{\prime}}\left[\begin{array}{c}
w^{(1)} \\
\vdots \\
w^{(M)}
\end{array}\right] \\
& =\left[\begin{array}{lll}
\left(A_{d}^{\prime}\right)^{M-1} & \ldots & I
\end{array}\right] \overline{B_{h d}^{\prime}} \phi_{M}
\end{aligned}
$$


where

$$
\begin{aligned}
A_{d}^{\prime} & :=\exp \left(A h^{\prime}\right) \\
B_{h d}^{\prime} & :=\left[\begin{array}{ll}
4 B_{0 d}^{\prime}-\frac{6}{h^{\prime}} B_{1 d}^{\prime} & -3 B_{0 d}^{\prime}+\frac{6}{h^{\prime}} B_{1 d}^{\prime}
\end{array}\right] \\
B_{0 d}^{\prime}: & \int_{0}^{h^{\prime}} \exp \left(A\left(h^{\prime}-\theta^{\prime}\right)\right) B_{1} d \theta^{\prime} \\
B_{1 d}^{\prime}: & : \int_{0}^{h^{\prime}} \exp \left(A\left(h^{\prime}-\theta^{\prime}\right)\right) \theta^{\prime} B_{1} d \theta^{\prime} \\
\phi_{M} & \left.:=\left[\phi_{0}^{(1)}\right)^{T}\left(\phi_{1}^{(1)}\right)^{T} \quad \ldots \quad\left(\phi_{0}^{(M)}\right)^{T}\left(\phi_{1}^{(M)}\right)^{T}\right]^{T} \\
\phi_{0}^{(i)} & :=\frac{1}{h^{\prime}} \int_{0}^{h^{\prime}} w^{(i)}\left(\tau^{\prime}\right) d \tau^{\prime}, \phi_{1}^{(i)}:=\frac{2}{\left(h^{\prime}\right)^{2}} \int_{0}^{h^{\prime}} w^{(i)}\left(\tau^{\prime}\right) \tau^{\prime} d \tau^{\prime}
\end{aligned}
$$

Hence, it is expected that considering the set of the vectors $\phi_{M}$ for all $w$ in the unit ball of $\left(L_{\infty}\left[0, h^{\prime}\right)\right)^{M n_{w}}$ and replacing $\mathbf{B}_{1} \mathbf{J}_{M 1}$ with the matrix in (47) may be helpful in computing $\left\|\mathcal{F}\left(\widehat{P}_{M 1}, \Psi\right)\right\|$. The following result is associated with $\mathbf{B}_{1}^{\prime} \mathbf{J}_{1}^{\prime}$ in (47) (or, more precisely, with each $\left[\left(\phi_{0}^{(i)}\right)^{T},\left(\phi_{1}^{(i)}\right)^{T}\right]^{T}$ in (52)) and plays a key role in representing the above vector set, denoted by $\boldsymbol{\Phi}_{M}$.

Theorem 3: Let

$$
\phi_{0}:=\frac{1}{h^{\prime}} \int_{0}^{h^{\prime}} w(t) d t, \quad \phi_{1}:=\frac{2}{\left(h^{\prime}\right)^{2}} \int_{0}^{h^{\prime}} w(t) t d t
$$

where $w \in L_{\infty}\left[0, h^{\prime}\right)$ is a scalar function. Then, the set of $\left(\phi_{0}, \phi_{1}\right)$ corresponding to all $w$ such that $\|w\| \leq 1$ is characterized by

$$
\begin{aligned}
-1 & \leq \phi_{0} \leq 1 \\
\frac{\phi_{0}^{2}+2 \phi_{0}-1}{2} & \leq \phi_{1} \leq \frac{-\phi_{0}^{2}+2 \phi_{0}+1}{2}
\end{aligned}
$$

Proof: It is obvious that (55) holds for every $w$ such that $\|w\| \leq 1$. Let us take an arbitrary $\phi_{0}$ satisfying (55), and take the following $w(t)$, which satisfies $\|w\| \leq 1$ and the first equation of (54).

$$
w(t)= \begin{cases}-1 & \left(0 \leq t<\frac{\left(1-\phi_{0}\right) h^{\prime}}{2}\right) \\ 1 & \left(\frac{\left(1-\phi_{0}\right) h^{\prime}}{2} \leq t<h^{\prime}\right)\end{cases}
$$

Because of a particular waveform of this $w$, together with the fact that $t$ is strictly increasing, it is obvious that this $w$ attains the maximum of $\phi_{1}$ among those satisfying the first equation of (54). Similarly, the above $w$ with $\phi_{0}$ replaced by $-\phi_{0}$ and with the sign inverted attains the minimum of $\phi_{1}$ among those satisfying the first equation of (54). For these particular $w$, we see that $\phi_{1}$ takes the extreme values in (56). Taking convex combinations of the above two extreme inputs completes the proof.

The area associated with (55) and (56) is shown in Fig. 2, which is obviously convex. Hence, it is expected that this area can be approximated in the $l_{\infty}$ sense, with an arbitrary degree of accuracy, by using the tangents for the boundary curves. This approximation procedure can be described as follows. We

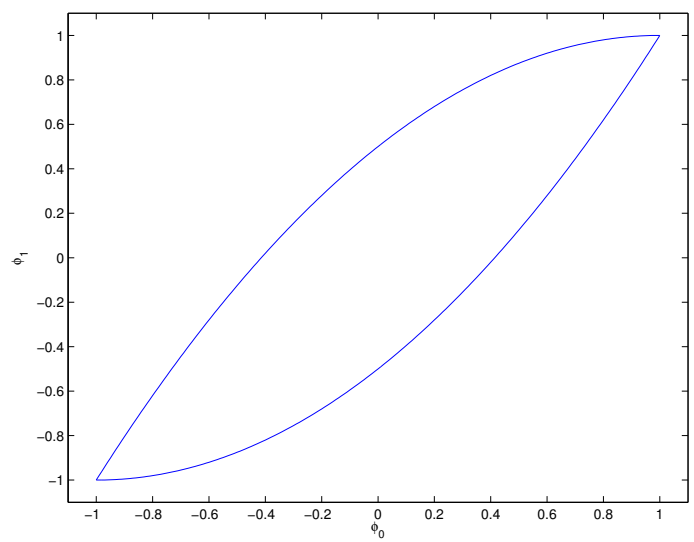

Fig. 2. The set of $\left(\phi_{0}, \phi_{1}\right)$ given by (54) with $\|w\| \leq 1$.

introduce the approximation parameter $N \in \mathbb{N}$, and draw the tangents for the boundary curves at the points $\phi_{0}=2(i-1) /$ $(N-1)-1$ for $i=1, \cdots, N(\geq 2)$ in Fig. 2. The area in Fig. 2 can then be approximated with these tangents by a convex polygon as shown in Fig. 3, and this technique can be applied $^{3}$ to the $l_{\infty}$ approximation of the set $\boldsymbol{\Phi}_{M}$. For example, when $N=3$ in Fig. 3, the convex polygon is determined by

$$
\begin{gathered}
-1 \leq \phi_{0} \leq 1 \\
-1 \leq-2 \phi_{0}+2 \phi_{1} \leq 1 \\
-1 \leq-2 \phi_{0}+\phi_{1} \leq 1
\end{gathered}
$$

and the set $\boldsymbol{\Phi}_{M}$ is outer-approximated with such $M 2 N$ polygons, or more precisely, by the set (denoted by $\boldsymbol{\Phi}_{M}^{[N]}$ ) of all vectors whose image by $\overline{\Delta^{[N]}}$ lies in the closed unit ball of $\mathbb{R}_{\infty}^{M N n_{w}}$, where

$$
\Delta^{[N]}=\Delta^{[3]}:=\left[\begin{array}{cc}
0_{n_{w}} & I_{n_{w}} \\
-2 I_{n_{w}} & 2 I_{n_{w}} \\
-2 I_{n_{w}} & I_{n_{w}}
\end{array}\right] \in \mathbb{R}_{\infty}^{N n_{w} \times 2 n_{w}}
$$

In the following discussions, it is convenient to describe the outer-approximated set $\boldsymbol{\Phi}_{M}^{[N]}$ as the unit ball image of $\overline{\Omega^{[N]}}$ with a suitable matrix $\Omega^{[N]}$. To give the representation of such $\Omega^{[N]}$, we take $v_{1}, \cdots, v_{N}$ to be the non-negative vector representations of the $N$ left-upper edges of the polygon, aligned from the rightmost one to the leftmost one ${ }^{4}$. For example, when $N=3$ in Fig. 3,

$$
v_{1}=\left[\begin{array}{c}
1 / 2 \\
0
\end{array}\right], v_{2}=\left[\begin{array}{l}
1 \\
1
\end{array}\right], v_{3}=\left[\begin{array}{c}
1 / 2 \\
1
\end{array}\right]
$$

By using these vectors $v_{i}=\left[v_{i 1}, v_{i 2}\right]^{T}$, the matrix $\Omega^{[N]}$ is defined as

$$
\Omega^{[N]}=\frac{1}{2}\left[\begin{array}{lll}
v_{11} I_{n_{w}} & \cdots & v_{N 1} I_{n_{w}} \\
v_{12} I_{n_{w}} & \cdots & v_{N 2} I_{n_{w}}
\end{array}\right] \in \mathbb{R}_{\infty}^{2 n_{w} \times N n_{w}}
$$

${ }^{3}$ We can apply Theorem 3 to each entry of the vector function $w$ because (53) can be computed entrywise. This leads to the formulation with the identity matrices $I_{n_{w}}$ in (61) and (63).

${ }^{4}$ We remark that the $i$ th block row of $\Delta^{[N]}$ corresponds, by definition, to the strip determined by two lines, each of which contains the edge corresponding to the vector $v_{i}$ and the other parallel edge. 


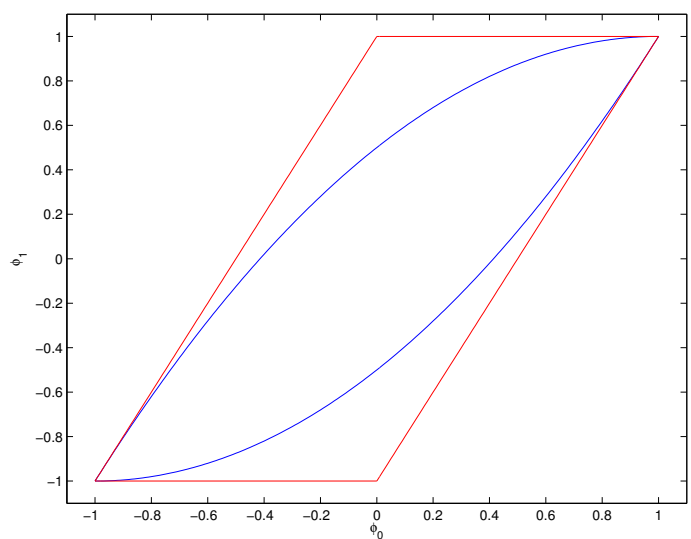

(a) $N=2$

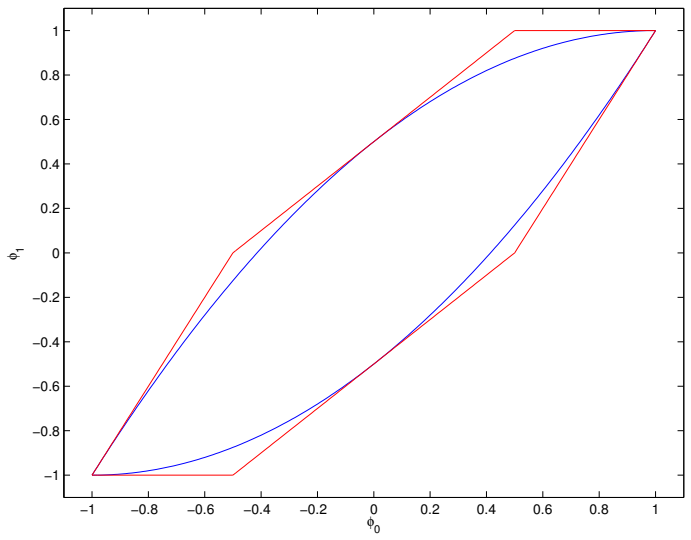

(b) $N=3$

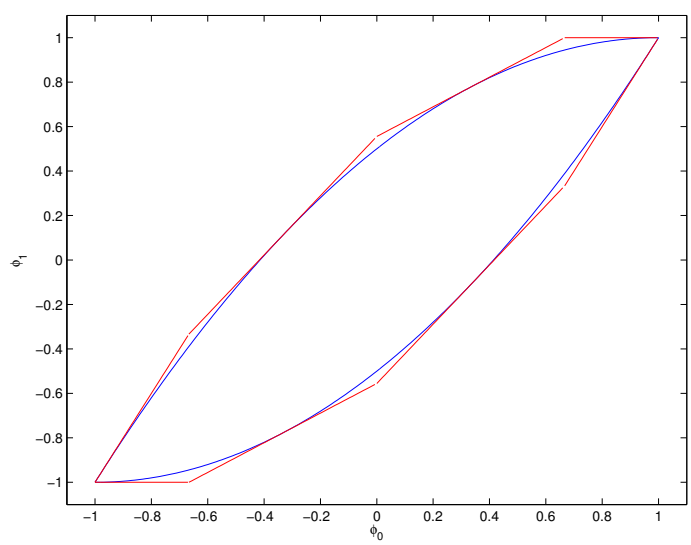

(c) $N=4$

Fig. 3. Approximation of $\phi_{0}$ and $\phi_{1}$.

Then, the outer-approximated set $\boldsymbol{\Phi}_{M}^{[N]}$ coincides with the set of all vectors $\overline{\Omega^{[N]}} w_{d}$ with $\left\|w_{d}\right\| \leq 1$ (see Appendix $\mathrm{C}$ for the proof). Summarizing the above arguments, we see from (47) that the operators $\mathbf{B}_{1}^{\prime} \mathbf{J}_{1}^{\prime}$ and $\mathbf{B}_{1} \mathbf{J}_{M 1}$ may respectively be replaced by the matrices

$$
B_{1}^{[N]^{\prime}}:=B_{h d}^{\prime} \Omega^{[N]}, B_{M 1}^{[N]}:=\left[\begin{array}{lll}
\left(A_{d}^{\prime}\right)^{M-1} & \cdots & I] \overline{B_{1}^{[N]^{\prime}}}
\end{array}\right.
$$

Furthermore, it is obvious from the above arguments that $\boldsymbol{\Phi}_{M}^{[N]}$ converges to $\boldsymbol{\Phi}_{M}$ as $N \rightarrow \infty$.

\section{B. Replacing $\mathbf{H}_{M 1} \mathbf{M}_{1}$ and $\mathbf{D}_{M 1}$ with appropriate matrices}

In this subsection, we give a method for replacing the operators $\mathbf{H}_{M 1} \mathbf{M}_{1}$ and $\mathbf{D}_{M 1}$ with appropriate matrices so that $\left\|\mathcal{F}\left(\widehat{P}_{M 1}, \Psi\right)\right\|$ can be computed as the $l_{\infty}$-induced norm of a discrete-time system (if the approximation error associated with the parameter $N$ in the preceding subsection were ignored; the error will be considered later).

Here, note that the infinite (Toeplitz) matrix representation of the input/output relation of $\mathcal{F}\left(\widehat{P}_{M 1}, \Psi\right)=$ $\mathbf{H}_{M 1} \mathbf{M}_{1} \mathcal{F}\left(P_{d}, \Psi\right) \mathbf{B}_{1} \mathbf{J}_{M 1}+\mathbf{D}_{M 1}$ is block lower triangular (with respect to the partitioning associated with $\widehat{w}_{k}$ and $\widehat{z}_{k}$ ) because of the structure of $P_{d}$, as mentioned in the preceding section. We then see that $\left\|\mathcal{F}\left(\widehat{P}_{M 1}, \Psi\right)\right\|$ coincides with the $L_{\infty}\left[0, h^{\prime}\right)$-induced norm of the "last block row" of the above infinite matrix. Note that $\mathcal{F}\left(\widehat{P}_{M 1}, \Psi\right)$ admits the representation

$$
\begin{cases}\xi_{k+1} & =\mathcal{A} \xi_{k}+J_{\Sigma} \mathbf{B}_{1} \mathbf{J}_{M 1} \widehat{w}_{k} \\ \widehat{z}_{k} & =\mathbf{H}_{M 1} \mathbf{M}_{1} C_{\Sigma} \xi_{k}+\mathbf{D}_{M 1} \widehat{z}_{k}\end{cases}
$$

where

$$
\begin{aligned}
& \xi_{k}:=\left[\begin{array}{ll}
x_{k}^{T} & \psi_{k}^{T}
\end{array}\right]^{T}, \\
& \mathcal{A}:=\left[\begin{array}{cc}
A_{d}+B_{2 d} D_{\Psi} C_{2 d} & B_{2 d} C_{\Psi} \\
B_{\Psi} C_{2 d} & A_{\Psi}
\end{array}\right], \\
& J_{\Sigma}:=\left[\begin{array}{l}
I \\
0
\end{array}\right] \in \mathbb{R}_{\infty}^{\left(n+n_{\Psi}\right) \times n}, \quad C_{\Sigma}:=\left[\begin{array}{cc}
I & 0 \\
D_{\Psi} C_{2 d} & C_{\Psi}
\end{array}\right]
\end{aligned}
$$

Hence, by the "last block row" mentioned above, we mean

$$
\begin{aligned}
\mathcal{P}_{M 1}:= & {\left[\begin{array}{llll}
\mathbf{D}_{M 1} & \mathbf{H}_{M 1} \mathbf{M}_{1} C_{\Sigma} J_{\Sigma} \mathbf{B}_{1} \mathbf{J}_{M 1} & \mathbf{H}_{M 1} \mathbf{M}_{1} C_{\Sigma} \mathcal{A} J_{\Sigma} \mathbf{B}_{1} \mathbf{J}_{M 1} \\
\mathbf{H}_{M 1} \mathbf{M}_{1} C_{\Sigma} \mathcal{A}^{2} J_{\Sigma} \mathbf{B}_{1} \mathbf{J}_{M 1} & \mathbf{H}_{M 1} \mathbf{M}_{1} C_{\Sigma} \mathcal{A}^{3} J_{\Sigma} \mathbf{B}_{1} \mathbf{J}_{M 1} & \cdots
\end{array}\right] }
\end{aligned}
$$

(where we have reversed the order of the operator entries for notational simplicity). Here, recall that $\mathbf{D}_{M 1}=$ $\overline{\mathbf{H}_{1}^{\prime}} \overline{\mathbf{M}_{1}^{\prime}} \Delta_{M}^{0} \overline{\mathbf{B}_{1}^{\prime}} \overline{\mathbf{J}_{1}^{\prime}}+\overline{\mathbf{D}_{p 1}^{\prime}}$, where $\Delta_{M}^{0}$ is strictly block lower triangular. Hence, it follows that $\overline{\mathbf{D}_{p 1}^{\prime}}$ can be handled separately from $\overline{\mathbf{H}_{1}^{\prime}} \overline{\mathbf{M}_{1}^{\prime}} \Delta_{M}^{0} \overline{\mathbf{B}_{1}^{\prime}} \overline{\mathbf{J}_{1}^{\prime}}$. Furthermore, to facilitate the treatment of the operator $\overline{\mathbf{D}_{p 1}^{\prime}}$, we introduce $\mathbf{D}_{p 10}^{\prime}:=\mathbf{D}_{p 1}^{\prime}-D_{11}$, which is an integral operator by the definition of $\mathbf{D}_{p 1}^{\prime}$ in (20). Then, by using the properties of $L_{\infty}\left[0, h^{\prime}\right), \mathcal{P}_{M 1}$ may be redefined as

$$
\begin{aligned}
\mathcal{P}_{M 1}:=\left[\begin{array}{lllll}
\overline{D_{11}} & \overline{\mathbf{D}_{p 10}^{\prime}} & \overline{\mathbf{H}_{1}^{\prime}} \overline{\mathbf{M}_{1}^{\prime}} \Delta_{M}^{0} \overline{\mathbf{B}_{1}^{\prime}} \overline{\mathbf{J}_{1}^{\prime}} & \mathbf{H}_{M 1} \mathbf{M}_{1} C_{\Sigma} J_{\Sigma} \mathbf{B}_{1} \mathbf{J}_{M 1} \\
\mathbf{H}_{M 1} \mathbf{M}_{1} C_{\Sigma} \mathcal{A} J_{\Sigma} \mathbf{B}_{1} \mathbf{J}_{M 1} & \mathbf{H}_{M 1} \mathbf{M}_{1} C_{\Sigma} \mathcal{A}^{2} J_{\Sigma} \mathbf{B}_{1} \mathbf{J}_{M 1} & \cdots
\end{array}\right]
\end{aligned}
$$

without changing its norm.

For simplicity, let us suppose $D_{11}=0$ for a while; we will return to the general case with $D_{11} \neq 0$ around the end of this subsection. Then, based on the property of $L_{\infty}\left[0, h^{\prime}\right)$, we can apply essentially the same arguments as those in [31] (which studies the computation of $\left\|\mathbf{D}_{11}\right\|$ ) to show that the input of the operator $\mathbf{D}_{p 10}^{\prime}$ may be restricted to a constant (vector) function in its treatment for the computation of $\left\|\mathcal{P}_{M 1}\right\|$ in (70); this is because $\mathbf{D}_{p 10}^{\prime}$ is simply an integral operator and the outputs of the operators in the other entries in $\mathcal{P}_{M 1}$ are linear functions. An immediate consequence of this restriction is that the output of $\mathcal{P}_{M 1}$ itself also becomes a linear function, so that the $L_{\infty}\left[0, h^{\prime}\right)$ norm of the output of $\mathcal{P}_{M 1}$ can be evaluated 
by considering only its values at $\theta^{\prime}=0$ and $\theta^{\prime}=h^{\prime}-0$. More precisely, since the operations of $\mathbf{H}_{M 1} \mathbf{M}_{1}$ and $\overline{\mathbf{D}_{p 10}^{\prime}}$ are respectively given by

$$
\begin{gathered}
\left(\mathbf{H}_{M 1} \mathbf{M}_{1}\left[\begin{array}{l}
x \\
u
\end{array}\right]\right)\left(\theta^{\prime}\right)=\overline{\mathbf{H}_{1}^{\prime}} \overline{\mathbf{M}_{1}^{\prime}}\left[\begin{array}{c}
I \\
\vdots \\
\left(A_{2 d}^{\prime}\right)^{M-1}
\end{array}\right]\left[\begin{array}{l}
x \\
u
\end{array}\right]\left(\theta^{\prime}\right) \\
=\overline{\left[C_{0}\left(I+A_{2} \theta^{\prime}\right)\right]}\left[\begin{array}{c}
I \\
\vdots \\
\left(A_{2 d}^{\prime}\right)^{M-1}
\end{array}\right]\left[\begin{array}{l}
x \\
u
\end{array}\right] \\
\overline{\left(\mathbf{D}_{p 10}^{\prime} w\right)}\left(\theta^{\prime}\right)=\overline{C_{1} B_{1} w(0) \theta^{\prime}}
\end{gathered}
$$

(where $w$ is assumed to be a constant function in (72) by the preceding arguments), the operators $\mathbf{H}_{1}^{\prime} \mathbf{M}_{1}^{\prime}$ and $\mathbf{D}_{p 10}^{\prime}$ in $\mathcal{P}_{M 1}$ may be replaced by the matrices

$$
\begin{aligned}
& H_{1}^{\prime}:=\left[\begin{array}{c}
C_{0} \\
C_{0}\left(I+A_{2} h^{\prime}\right)
\end{array}\right] \in \mathbb{R}_{\infty}^{2 n_{z} \times n}, \\
& D_{p 10}^{\prime}:=\left[\begin{array}{c}
0 \\
C_{1} B_{1} h^{\prime}
\end{array}\right] \in \mathbb{R}_{\infty}^{2 n_{z} \times n_{w}}
\end{aligned}
$$

respectively, without changing $\left\|\mathcal{P}_{M 1}\right\|$. After such replacement, the only operators remaining in $\mathcal{P}_{M 1}$ are $\mathbf{B}_{1} \mathbf{J}_{M 1}$, but the preceding arguments suggest that it can be further replaced by the matrix $B_{M 1}^{[N]}$ in (64).

By using the matrices $B_{1}^{[N]^{\prime}}, B_{M 1}^{[N]}, H_{1}^{\prime}$ and $D_{p 10}^{\prime}$, the $L_{\infty}\left[0, h^{\prime}\right)$-induced norm $\left\|\mathcal{P}_{M 1}\right\|$ and thus $\left\|\mathcal{F}\left(\widehat{P}_{M 1}, \Psi\right)\right\|$ is obtained by computing the $l_{\infty}$-induced norm of the matrix $P_{M 1}^{[N]}$ (with an error associated with the approximation of $\boldsymbol{\Phi}_{M}$ by $\left.\boldsymbol{\Phi}_{M}^{[N]}\right)^{5}$ given by

$$
\begin{aligned}
P_{M 1}^{[N]}:= & {\left[\begin{array}{lllll}
\overline{\widetilde{D}_{11}} & \overline{D_{p 10}^{\prime}} & \overline{H_{1}^{\prime}} \Delta_{M}^{0} & \overline{B_{1}^{[N]^{\prime}}} & H_{M 1} C_{\Sigma} J_{\Sigma} B_{M 1}^{[N]} \\
& H_{M 1} C_{\Sigma} \mathcal{A} J_{\Sigma} B_{M 1}^{[N]} & H_{M 1} C_{\Sigma} \mathcal{A}^{2} J_{\Sigma} B_{M 1}^{[N]} & \cdots
\end{array}\right] }
\end{aligned}
$$

where

$$
H_{M 1}:=\overline{H_{1}^{\prime}}\left[\begin{array}{c}
I \\
\vdots \\
\left(A_{2 d}^{\prime}\right)^{M-1}
\end{array}\right], \widetilde{D}_{11}:=\left[\begin{array}{c}
D_{11} \\
D_{11}
\end{array}\right]
$$

Here, we note again that $\Delta_{M}^{0}$ is strictly block lower triangular and $N \geq 2$. Then, based on the property of $L_{\infty}\left[0, h^{\prime}\right)$, the matrix $\bar{P}_{M 1}^{[N]}$ may be redefined as

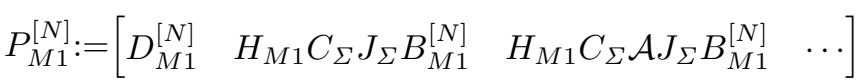

without changing its norm, with the matrices

$$
\begin{aligned}
& D_{M 1}^{[N]}:=\overline{H_{1}^{\prime}} \Delta_{M}^{0} \overline{B_{1}^{[N]^{\prime}}}+\overline{D_{p 1}^{[N]^{\prime}}} \in \mathbb{R}_{\infty}^{2 M n_{z} \times M N n_{w}} \\
& D_{p 1}^{[N]^{]^{\prime}}}:=\left[\begin{array}{lll}
D_{p 10}^{\prime} & \widetilde{D}_{11} & 0
\end{array}\right] \in \mathbb{R}_{\infty}^{2 n_{z} \times N n_{w}}
\end{aligned}
$$

Because the matrix $P_{M 1}^{[N]}$ (in the modified form in (77)) corresponds to the "last block row" of the input/output relation of a discrete-time system, the $L_{1}$ problem of $\mathcal{F}\left(\widehat{P}_{M 1}, \Psi\right)$ is reducible to the discrete-time $l_{1}$ problem.

\footnotetext{
${ }^{5}$ Because $\boldsymbol{\Phi}_{M}^{[N]}$ converges to $\boldsymbol{\Phi}_{M}$ as $N \rightarrow \infty$, we can have an arbitrary degree of accuracy. See Theorem 4.
}

Remark 8: We have recovered the general case with $D_{11} \neq 0$ in (75) and thereafter. Indeed, the preceding arguments immediately lead to (75) with the first entry $\overline{\widetilde{D}_{11}}$ removed, and it is not hard to see that dealing with $D_{11} \neq 0$ leads to (75) as it is; the treatment of $D_{11}$ is essentially the same as that in [10].

\section{Discretization of the continuous-time generalized plant}

This subsection is devoted to showing that the arguments in the preceding subsection immediately lead us to a discretization procedure for the continuous-time generalized plant $P$ that can be used in dealing with the $L_{1}$ problem of $\mathcal{F}\left(\widehat{P}_{M 1}, \Psi\right)$ through a discrete-time $l_{1}$ problem.

Indeed, let us consider the discrete-time plant given by

$$
P_{M 1 d}^{[N]}:\left\{\begin{array}{l}
x_{k+1}=A_{d} x_{k}+B_{M 1}^{[N]} w_{k}+B_{2 d} u_{k} \\
z_{k}=C_{M 1} x_{k}+D_{M 1}^{[N]} w_{k}+D_{M 2} u_{k} \\
y_{k}=C_{2 d} x_{k}
\end{array}\right.
$$

where the matrices $C_{M 1} \in \mathbb{R}_{\infty}^{2 M n_{z} \times n}$ and $D_{M 2} \in \mathbb{R}_{\infty}^{2 M n_{z} \times n_{u}}$ are given by

$$
\left[\begin{array}{ll}
C_{M 1} & D_{M 2}
\end{array}\right]:=H_{M 1}
$$

The other matrices are given in (4), (64) and (78). Then, we readily see that the closed-loop system obtained by connecting $\Psi$ to the above discrete-time plant has the state-space representation $\left(\mathcal{A}, J_{\Sigma} B_{M 1}^{[N]}, H_{M 1} C_{\Sigma}, D_{M 1}^{[N]}\right)$. Since the "last block row" of the infinite (Toeplitz) matrix representation of the input/output relation of this closed-loop system (with the entries aligned in the reverse order) is nothing but $P_{M 1}^{[N]}$ given in (77), it follows readily that computing $\left\|P_{M 1}^{[N]}\right\|$ is equivalent to computing the $l_{\infty}$-induced norm of the above closed-loop system. This implies that the $L_{1}$ problem of $\mathcal{F}\left(\widehat{P}_{M 1}, \Psi\right)$ with the relevant outer-approximation of $\boldsymbol{\Phi}_{M}$ by $\boldsymbol{\Phi}_{M}^{[N]}$ is exactly reducible to the discrete-time $l_{1}$ problem for the discretized generalized plant $P_{M 1 d}^{[N]}$.

Let us denote by $\left\|\mathcal{F}\left(P_{M 1 d}^{[N]}, \Psi\right)\right\|$ the $l_{\infty}$-induced norm computed through this outer-approximation. Then, by the preceding arguments, $\left\|\mathcal{F}\left(P_{M 1 d}^{[N]}, \Psi\right)\right\|$ can be made arbitrarily close to $\left\|\mathcal{F}\left(\widehat{P}_{M 1}, \Psi\right)\right\|$ by taking a sufficiently large $N$. More precisely, we have the following result regarding the approximation of $\|\mathcal{F}(\widehat{P}, \Psi)\|$ by $\left\|\mathcal{F}\left(P_{M 1 d}^{[N]}, \Psi\right)\right\|$, where $\kappa^{[N]}$ is defined as the largest $\kappa>0$ such that $\kappa \boldsymbol{\Phi}_{M}^{[N]} \subset \boldsymbol{\Phi}_{M}$ (which can be computed easily for each $N$ ).

Theorem 4: The inequality

$$
\begin{aligned}
\left(1-\frac{K_{0}}{M^{2}}\right) & \|\mathcal{F}(\widehat{P}, \Psi)\|-\frac{K_{1}}{M^{2}} \leq\left\|\mathcal{F}\left(P_{M 1 d}^{[N]}, \Psi\right)\right\| \\
\leq & \frac{1}{\kappa^{[N]}}\left[\left(1+\frac{K_{0}}{M^{2}}\right)\|\mathcal{F}(\widehat{P}, \Psi)\|+\frac{K_{1}}{M^{2}}\right]
\end{aligned}
$$

holds and $\kappa^{[N]}$ converges to 1 as $N \rightarrow \infty$.

Note that the above lower bound of $\left\|\mathcal{F}\left(P_{M 1 d}^{[N]}, \Psi\right)\right\|$ is the same as that of $\left\|\mathcal{F}\left(\widehat{P}_{M 1}, \Psi\right)\right\|$ in (40), while the upper bound is larger than that of $\left\|\mathcal{F}\left(\widehat{P}_{M 1}, \Psi\right)\right\|$ in (40) by the factor of $1 / \kappa^{[N]}$; this follows immediately from $\boldsymbol{\Phi}_{M} \subset \boldsymbol{\Phi}_{M}^{[N]}$ and $\kappa^{[N]} \boldsymbol{\Phi}_{M}^{[N]} \subset \boldsymbol{\Phi}_{M}$. The assertion on $\kappa^{[N]}$ is also obvious (see 
Fig. 3). This theorem obviously ensures that the $L_{1}$ optimal controller synthesis can be carried out through $\mathcal{F}\left(P_{M 1 d}^{[N]}, \Psi\right)$ with sufficiently large $M$ and $N$.

Remark 9: As suggested in Remarks 6 and 7, our arguments adapted to FSFH approximation can lead to the inequality

$$
\left(1-\frac{T_{0}}{M}\right)\|\mathcal{F}(\widehat{P}, \Psi)\|-\frac{T_{1}}{M} \leq\left\|\mathcal{F}\left(P_{M 0 d}, \Psi\right)\right\| \leq\|\mathcal{F}(\widehat{P}, \Psi)\|
$$

with the same discretized generalized plant $P_{M 0 d}$ as that in the existing studies [8]-[10] on the FSFH approximation approach. This is an FSFH approximation counterpart of (82), where explicit values of $T_{0}$ and $T_{1}$ (see Appendix B), independent of the controller $\Psi$, can be determined in a similar way to the case of piecewise linear approximation. This leads to quantitative improvement over [9] for the evaluation of $T_{0}$ and $T_{1}$, but its qualitative implication is nothing beyond what has been established in [9]; it justifies the assertion that the FSFH approximation approach results in an approximate method for designing $\Psi$ by solving the $l_{1}$ optimal control problem of minimizing $\left\|\mathcal{F}\left(P_{M 0 d}, \Psi\right)\right\|$.

\section{NUMERICAL EXAMPLE}

This section examines the effectiveness of the discretization method developed in this paper through a numerical example.

We consider the direct current (DC) motor shown in Figure 4 , where $u$ is the input voltage, $y=z$ is the rotational angle, and $w$ is the bounded persistent disturbance affecting the rotational speed. The purpose in this example is to design the discrete-time controller $\Psi$, which measures the rotational angle $y$ and manipulates the input voltage $u$, that minimizes the effect of the bounded persistent disturbance $w$ on the rotational angle $z$ (i.e., the $L_{1}$ optimal sampled-data controller synthesis for minimizing the $L_{\infty}$-induced norm from $w$ to $z$ ). The generalized plant $P$ in Figure 4 can be described by the following state-space representation.

$$
\begin{aligned}
& A=\left[\begin{array}{ll}
1 & -1 \\
2 & -2
\end{array}\right], \quad B_{1}=\left[\begin{array}{c}
1 \\
-1
\end{array}\right], \quad B_{2}=\left[\begin{array}{c}
0 \\
-1
\end{array}\right] \\
& C_{1}=\left[\begin{array}{ll}
1 & 0
\end{array}\right], C_{2}=\left[\begin{array}{ll}
1 & 0
\end{array}\right], D_{11}=D_{12}=0
\end{aligned}
$$

We take the sampling period $h=0.3,0.15$ and 0.05 , and discrete-time controller synthesis is carried out for each of these sampling periods. Let $\Psi_{M 0}$ and $\Psi_{M 1}^{[N]}$ be the discretetime $l_{1}$ optimal controllers minimizing respectively the $l_{\infty}$ induced norm $\left\|\mathcal{F}\left(P_{M 0 d}, \Psi\right)\right\|$ through the $L_{1}$-discretization $P_{M 0 d}$ of $P$ via FSFH approximation (as mentioned in Remark 9) and $\left\|\mathcal{F}\left(P_{M 1 d}^{[N]}, \Psi\right)\right\|$ through the $L_{1}$-discretization

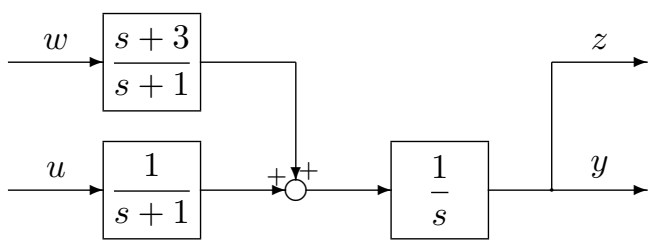

Fig. 4. DC motor with bounded persistent disturbances.
$P_{M 1 d}^{[N]}$ of $P$ via the piecewise linear approximation approach. They are designed under the FSFH/fast-lifting parameter $M=$ $2,3,4$ together with $N=3$.

We are mainly interested in the comparison between the existing FSFH approximation approach and the piecewise linear approximation approach developed in this paper. Hence, the following tables consist of Case (a) for the existing approach and Case (b) for the new approach. Regarding $\Psi_{M 0}$ with sampling period $h=0.3$, the results of the $l_{\infty}$-induced norm of the discrete-time system $\mathcal{F}\left(P_{M 0 d}, \Psi_{M 0}\right)$ and the $L_{\infty^{-}}$ induced norm of the sampled-data system $\Sigma_{\mathrm{SD}}$ with $\Psi=\Psi_{M 0}$ (i.e., $\left.\left\|\mathcal{F}\left(\widehat{P}, \Psi_{M 0}\right)\right\|\right)$ are shown in Table III(a). Similarly, regarding $\Psi_{M 1}^{[N]}$ for the same $h$, the $l_{\infty}$-induced norm of the discrete-time system $\mathcal{F}\left(P_{M 1 d}^{[N]}, \Psi_{M 1}^{[N]}\right)$ and the $L_{\infty}$-induced norm of the sampled-data system $\Sigma_{\mathrm{SD}}$ with $\Psi=\Psi_{M 1}^{[N]}$ (i.e., $\left.\left\|\mathcal{F}\left(\widehat{P}, \Psi_{M 1}^{[N]}\right)\right\|\right)$ are shown in Table III(b). Furthermore, the results relevant to the sampling period $h=0.15$ and $h=0.05$ are shown in Tables IV and V, respectively. These values are computed so that they are accurate up to the digits shown therein ${ }^{6}$.

We can see from these tables that $\Psi_{M 1}^{[N]}$ succeeds in drastically reducing the $L_{\infty}$-induced norm of the closed-loop sampled-data system compared with $\Psi_{M 0}$ under the same approximation parameter $M$ and sampling period $h$. This could be interpreted as that the piecewise linear approximation approach developed in this paper achieves better performance than the conventional FSFH approximation approach in the $L_{1}$ optimal sampled-data controller synthesis.

Furthermore, we can also see from these tables that the ratio $r_{1}:=\left\|\mathcal{F}\left(\widehat{P}, \Psi_{M 1}^{[N]}\right)\right\| /\left\|\mathcal{F}\left(P_{M 1 d}^{[N]}, \Psi_{M 1}^{[N]}\right)\right\|$ is much closer to 1 than $r_{0}:=\left\|\mathcal{F}\left(\widehat{P}, \Psi_{M 0}\right)\right\| /\left\|\mathcal{F}\left(P_{M 0 d}, \Psi_{M 0}\right)\right\|$ under the same $M$ and $h$. This implies that taking the intersample behavior of the sampled-data system $\Sigma_{\mathrm{SD}}$ into account can be more accurately reflected on the controller design, even with a relatively small $M$, through the piecewise linear approximation than through the FSFH approximation.

Furthermore, in regard to the above two ratios, we note that the ratio $r_{0}$ for the FSFH approximation becomes quite large as the sampling period becomes smaller. This implies that the FSFH approximation technique with a relatively small $M$ tends to lose its rationale in the $L_{1}$ optimal sampled-data controller synthesis as the sampling period becomes smaller (while taking a large $M$ is unrealistic due to the increase in the scale of the associated computations in the controller synthesis).

Such an observation would be quite counterintuitive because the FSFH approximation technique is generally believed to work more adequately even with a small $M$ as the sampling period becomes smaller. In this regard, it is worthwhile to note that the same tendency can be observed with respect to the ratio $r_{1}$ for the piecewise linear approximation (but with much milder discrepancy from 1 than $r_{0}$ even when $h$ becomes

\footnotetext{
${ }^{6}$ Even though the arguments of Theorem 4 can be used also for analysis, the $L_{\infty}$-induced norm computation for the designed controllers is carried out through the arguments in [30]. This is because the computation of the upper and lower bounds therein is much simpler, even though its extension to controller synthesis is very hard.
} 
TABLE III

RESULTS OF THE $L_{1}$ OPTIMAL CONTROLLER SYNTHESIS IN THE SAMPLING PERIOD $h=0.3$.

Case (a): FSFH approximation approach

\begin{tabular}{c|ccc}
\hline$M$ & 2 & 3 & 4 \\
\hline$\left\|\mathcal{F}\left(P_{M 0 d}, \Psi_{M 0}\right)\right\|$ & 0.4835 & 0.5362 & 0.5552 \\
$\left\|\mathcal{F}\left(\widehat{P}, \Psi_{M 0}\right)\right\|$ & 3.7012 & 1.7814 & 1.6169 \\
\hline
\end{tabular}

Case (b): Piecewise linear approximation approach

\begin{tabular}{c|ccc}
\hline$M$ & 2 & 3 & 4 \\
\hline$\left\|\mathcal{F}\left(P_{M 1 d}^{[N]}, \Psi_{M}^{[N]}\right)\right\|$ & 0.6617 & 0.6226 & 0.5961 \\
$\left\|\mathcal{F}\left(\widehat{P}, \Psi_{M 1}^{[N]}\right)\right\|$ & 1.5048 & 0.6453 & 0.6351 \\
\hline
\end{tabular}

TABLE IV

RESULTS OF THE $L_{1}$ OPTIMAL CONTROLLER SYNTHESIS IN THE SAMPLING PERIOD $h=0.15$.

Case (a): FSFH approximation approach

\begin{tabular}{c|ccc}
\hline$M$ & 2 & 3 & 4 \\
\hline$\left\|\mathcal{F}\left(P_{M 0 d}, \Psi_{M 0}\right)\right\|$ & 0.2114 & 0.2348 & 0.2426 \\
$\left\|\mathcal{F}\left(\widehat{P}, \Psi_{M 0}\right)\right\|$ & 2.9118 & 1.2624 & 0.9629 \\
\hline
\end{tabular}

Case (b): Piecewise linear approximation approach

\begin{tabular}{c|ccc}
\hline$M$ & 2 & 3 & 4 \\
\hline$\left\|\mathcal{F}\left(P_{M]}^{[N]}, \Psi_{M 1}^{[N]}\right)\right\|$ & 0.2859 & 0.2715 & 0.2623 \\
$\left\|\mathcal{F}\left(\widehat{P}, \Psi_{M 1}^{[N]}\right)\right\|$ & 0.9915 & 0.7046 & 0.3047 \\
\hline
\end{tabular}

TABLE V

RESULTS OF THE $L_{1}$ OPTIMAL CONTROLLER SYNTHESIS IN THE SAMPLING PERIOD $h=0.05$.

Case (a): FSFH approximation approach

\begin{tabular}{c|ccc}
\hline$M$ & 2 & 3 & 4 \\
\hline$\left\|\mathcal{F}\left(P_{M 0 d}, \Psi_{M 0}\right)\right\|$ & 0.0635 & 0.0704 & 0.0728 \\
$\left\|\mathcal{F}\left(\widehat{P}, \Psi_{M 0}\right)\right\|$ & 2.3753 & 0.9385 & 0.6412 \\
\hline
\end{tabular}

Case (b): Piecewise linear approximation approach

\begin{tabular}{c|ccc}
\hline$M$ & 2 & 3 & 4 \\
\hline$\left\|\mathcal{F}\left(P_{M 1}^{[N]}, \Psi_{M 1}^{[N]}\right)\right\|$ & 0.0849 & 0.0807 & 0.0790 \\
$\left\|\mathcal{F}\left(\widehat{P}, \Psi_{M 1}^{[N]}\right)\right\|$ & 0.3884 & 0.1373 & 0.1107 \\
\hline
\end{tabular}

smaller). Hence, we could interpret that the $L_{1}$ optimal controller synthesis problem possesses an intrinsic feature that is in sharp contrast with a general (ungrounded) understanding or other well-studied problems (such as stability, $H_{2}$ and $H_{\infty}$ problems) of sampled-data systems; for the latter problems, we could usually expect in a rough sense that for sufficiently small $h$, the effect of the intersample behavior would be rather small and whatever sort of approximate treatment could suffice.

A possible interpretation behind such a distinct and counterintuitive nature of the $L_{1}$ optimal sampled-data controller synthesis problem might be deeply related with the feature of the functions in $L_{\infty}$; the worst input for this problem might be a such one that behaves rather oddly with many discontinuities, for which piecewise constant approximation may not be powerful enough. Indeed, we have confirmed the above inference in the present example by computing the (approximately-)worst input under the designed controllers by exploiting the arguments in [30]; as the optimal controller tends to have a larger gain as the sampling period decreases, the worst input tends to have discontinuities more frequently, and this prevents the FSFH approximation from accordingly becoming more suitable, unlike one's naive intuition (that could work if the worst input is smooth and irrelevant with the sampling period).

For such a difficult $L_{1}$ problem among the problems for sampled-data systems, it is quite prominent that the ratio $r_{1}$ is much improved over the ratio $r_{0}$ in terms of their discrepancies from 1, regardless of the sampling period. These observations undoubtedly demonstrate the drastically outperforming ability of the synthesis method developed with our piecewise linear approximation treatment over the conventional method through the FSFH approximation treatment. Facilitating design of effective controllers through such drastic improvement by our piecewise linear approximation approach is believed to shed renewed light on the application of sampled-data $L_{1}$ optimal control to practical problems.

In this regard, we would like to remark that other conventional discretization methods such as Euler and bilinear transformations cannot ensure closed-loop stability and indeed failed to attain stability in the example here when the discretetime $l_{1}$ optimal controllers for the generalized plant discretized with these conventional methods were employed. We believe that the loss of closed-loop stability in these conventional discretization methods even with a small sampling period again reflects the intrinsic difficulties of the $L_{1}$ optimal sampled-data controller synthesis problem; because of the peculiar nature of the worst input, the conventional discretization methods cannot simply be expected to work fine while maintaining closed-loop stability for this particular type of sampled-data controller synthesis problem.

\section{CONCLUSION}

In this paper, we developed a new discretization method for the $L_{1}$ optimal controller synthesis problem of sampled-data systems by using an idea of piecewise linear approximation. The key techniques in this development were the application of fast-lifting and the introduction of two types of 'linearizing operators,' one for input signals and the other for output signals. With these approximation operators, the continuoustime signals (i.e., the input signals and the output signals) were approximated by piecewise linear functions, and this led to the operator approximation of the sampled-data mapping $\mathcal{F}(\widehat{P}, \Psi)$.

To demonstrate the benefit of this operator approximation in the $L_{1}$ optimal controller synthesis problem of sampled-data systems, we first established Theorem 2 or the inequality (40) through the arguments of preadjoint operators. This inequality was shown to play an important role in the piecewise linear approximation treatment for the $L_{1}$ optimal controller synthesis problem of sampled-data systems. In particular, it was shown that the convergence rate associated with the piecewise linear approximation is $1 / M^{2}$ with respect to the fast-lifting parameter $M$, in contrast to that given by $1 / M$ in the FSFH (i.e., piecewise constant) approximation method with respect to the FSFH parameter $M$ (which plays essentially the same role as the fast-lifting parameter $M$ ). We then provided a discretization procedure for the continuous-time generalized plant through the piecewise linear approximation. This procedure enables us to give an 'approximately equivalent' discrete-time generalized plant to the continuous-time generalized plant with any degree of accuracy, and thus the $L_{1}$ optimal controller synthesis problem of sampled-data systems can be reduced to 
the discrete-time $l_{1}$ optimal controller synthesis problem in a more effective way than the conventional method through the FSFH approximation. Finally, we examined effectiveness of the developed method through a numerical study, and it was confirmed that the piecewise linear approximation method works far more effectively than the conventional method.

In connection with this numerical study, we also discussed a distinct and somewhat counterintuitive nature of the $L_{1}$ optimal sampled-data controller synthesis problem; it may be in sharp contrast with such well-studied problems as the $\mathrm{H}_{2}$ and $H_{\infty}$ problems of sampled-data systems, and strongly supports the necessity of a sophisticated method in the $L_{1}$ synthesis problem of sampled-data systems, particularly our piecewise linear approximation approach. The success in this paper in the establishment of such a sophisticated method is believed to contribute to wider applications of sampled-data $L_{1}$ optimal control to practical problems.

\section{ACKNOWLEDGMENT}

The authors would like to thank Yoshito Ohta for his comments on the $L_{1}$ optimal control problem. The $L_{1}$ optimal controller synthesis in the numerical example was carried out by the software developed by I. J. Diaz-Bobillo and M. A. Dahleh, to whom the present authors are very grateful.

\section{APPENDIX}

\section{A. Proof of Lemma 1}

In this appendix, we give a brief proof of Lemma 1 .

a) As a preliminary step to obtain the bound (29), we first show the existence of a constant $c_{1}$ independent of $M$ such that

$$
h\|x\|_{1} \leq c_{1}\left\|\mathbf{B}_{1 *} x\right\|_{1}, \quad \forall x \in \mathbb{R}_{1}^{n}
$$

To show this, let us introduce the operator $\mathbf{T}_{B}$ : $\left(L_{1}[0, h)\right)^{n_{w}} \rightarrow \mathbb{R}_{1}^{n}$ given by

$$
\mathbf{T}_{B} w=\int_{0}^{h} \exp (A(h-\theta)) B_{1} w(\theta) d \theta
$$

(Note that $\mathbf{T}_{B}$ is formally the same as $\mathbf{B}_{1}$ except that the domain is not $\left(L_{\infty}[0, h)\right)^{n_{w}}$ but $\left(L_{1}[0, h)\right)^{n_{w}}$ and the codomain is not $\mathbb{R}_{\infty}^{n}$ but $\mathbb{R}_{1}^{n}$.) Then, since $\mathbf{B}_{1 *}$ is given by $\left(\mathbf{B}_{1 *} x\right)(\theta)=B_{1}^{T} \exp \left(A^{T}(h-\theta)\right) x$, we have

$$
\mathbf{T}_{B} \mathbf{B}_{1 *}=\int_{0}^{h} \exp (A(h-\theta)) B_{1} B_{1}^{T} \exp \left(A^{T}(h-\theta)\right) d \theta
$$

Note that (87) implies that $\mathbf{T}_{B} \mathbf{B}_{1 *}$ coincides with the controllability Grammian, which we denote by $W_{B}$. We then have

$$
\begin{gathered}
h\|x\|_{1}=h\left\|W_{B}^{-1} \mathbf{T}_{B} \mathbf{B}_{1 *} x\right\|_{1} \leq h\left\|W_{B}^{-1}\right\|_{1}\left\|\mathbf{T}_{B}\right\|_{1}\left\|\mathbf{B}_{1 *} x\right\|_{1} \\
\leq h\left\|W_{B}^{-1}\right\|_{1} e^{\|A\|_{1} h}\left\|B_{1}\right\|_{1}\left\|\mathbf{B}_{1 *} x\right\|_{1}=: c_{1}\left\|\mathbf{B}_{1 *} x\right\|_{1}
\end{gathered}
$$

because $W_{B}^{-1}$ exists by the controllability assumption.

Next, let $g \in R\left(\mathbf{B}_{1 *}\right)$, i.e., $g(\theta)=B_{1}^{T} \exp \left(A^{T}(h-\theta)\right) x$ for some $x \in \mathbb{R}_{1}^{n}$. Then, a direct computation with (31) and (32) leads to

$$
\left(\mathbf{J}_{M 1 *} g\right)\left(\theta^{\prime}\right)=\left[\begin{array}{c}
B_{1}^{T}\left(A_{d T}^{\prime}\right)^{M-1} f\left(A, \theta^{\prime}\right)^{T} \\
\vdots \\
B_{1}^{T} f\left(A, \theta^{\prime}\right)^{T}
\end{array}\right] x
$$

where

$$
\begin{aligned}
& A_{d T}^{\prime}:=\left(A_{d}^{\prime}\right)^{T} \\
& f\left(A, \theta^{\prime}\right):=f_{0}\left(\theta^{\prime}\right) A_{0 d}^{\prime}+f_{1}\left(\theta^{\prime}\right) A_{1 d}^{\prime} \\
& A_{0 d}^{\prime}:=\int_{0}^{h^{\prime}} \exp \left(A\left(h^{\prime}-\tau^{\prime}\right)\right) d \tau^{\prime} \\
& A_{1 d}^{\prime}:=\int_{0}^{h^{\prime}} \exp \left(A\left(h^{\prime}-\tau^{\prime}\right)\right) \tau^{\prime} d \tau^{\prime}
\end{aligned}
$$

Because $h\|x\|_{1} \leq c_{1}\|g\|_{1}$ by (85), a direct computation together with the Taylor expansion arguments leads to

$$
\begin{aligned}
& \left\|\left(\mathbf{L}_{M}-\mathbf{J}_{M 1 *}\right) g\right\|_{1}=\left\|\mathbf{L}_{M} \mathbf{B}_{1 *} x-\mathbf{J}_{M 1 *} \mathbf{B}_{1 *} x\right\|_{1} \\
& \leq\left\|\left(I-\overline{\mathbf{J}_{1 *}^{\prime}}\right) \mathbf{L}_{M} \mathbf{B}_{1 *} x\right\|_{1} \\
& \leq\left\|B_{1}^{T}\right\|_{1} e^{\left\|A^{T}\right\|_{1} h} M \\
& \quad \cdot \int_{0}^{h^{\prime}}\left\|f\left(A, \theta^{\prime}\right)^{T}-\exp \left(A^{T}\left(h^{\prime}-\theta^{\prime}\right)\right)\right\|_{1} d \theta^{\prime}\|x\|_{1} \\
& \leq\left\|B_{1}^{T}\right\|_{1} e^{\left\|A^{T}\right\|_{1} h} M \frac{1}{2} \frac{h^{3}}{M^{3}}\left\|A_{d T}^{\prime}\right\|_{1} \cdot\left\|A^{T}\right\|_{1}^{2} e^{\left\|A^{T}\right\|_{1} h^{\prime}}\|x\|_{1} \\
& \leq \frac{h^{2}}{2 M^{2}} e^{3\|A\| h}\|A\|^{2}\left\|B_{1}\right\| c_{1}\|g\|_{1}=: \frac{K_{B}}{M^{2}}\|g\|_{1}
\end{aligned}
$$

This implies (29) and the proof of part a) is completed.

b) As a preliminary step to obtain the bound (30), we show the existence of a constant $c_{\infty}$ independent of $M$ such that

$$
\|p\| \leq c_{\infty}\left\|\mathbf{M}_{1} p\right\|, \quad \forall p \in \mathbb{R}_{\infty}^{n+n_{u}}
$$

To show this, we introduce the operator $\mathbf{T}_{C}:\left(L_{\infty}[0, h)\right)^{n_{z}} \rightarrow$ $\mathbb{R}_{\infty}^{n+n_{u}}$ given by

$$
\mathbf{T}_{C} z=\int_{0}^{h} \exp \left(A_{2}^{T} \theta\right) C_{0}^{T} z(\theta) d \theta
$$

Then, we have

$$
\mathbf{T}_{C} \mathbf{M}_{1}=\int_{0}^{h} \exp \left(A_{2}^{T} \theta\right) C_{0}^{T} C_{0} \exp \left(A_{2} \theta\right) d \theta=: W_{C}
$$

Since $W_{C}$ is the observability Grammian, the remaining part of the proof proceeds in a similar way to part a) with $\|\cdot\|_{1}$ replaced by $\|\cdot\|$; by using essentially the same arguments as part a), we have

$$
\begin{gathered}
\|p\|=\left\|W_{C}^{-1} \mathbf{T}_{C} \mathbf{M}_{1} p\right\| \leq\left\|W_{C}^{-1}\right\| \cdot\left\|\mathbf{T}_{C}\right\| \cdot\left\|\mathbf{M}_{1} p\right\| \\
\leq\left\|W_{C}^{-1}\right\| h e^{\left\|A_{2}^{T}\right\| h}\left\|C_{0}^{T}\right\| \cdot\left\|\mathbf{M}_{1} p\right\|=: c_{\infty}\left\|\mathbf{M}_{1} p\right\|
\end{gathered}
$$

From (95) and a direct computation together with the Taylor expansion arguments, we can obtain

$$
\begin{aligned}
& \left\|\left.\left(\mathbf{L}_{M}-\mathbf{H}_{M 1}\right)\right|_{R\left(\mathbf{M}_{1}\right)}\right\| \\
\leq & \frac{h^{2}}{2 M^{2}}\left\|C_{0}\right\| \cdot\left\|A_{2}\right\|^{2} e^{2\left\|A_{2}\right\| h} c_{\infty}=: \frac{K_{C}}{M^{2}}
\end{aligned}
$$

\section{B. $T_{0}$ and $T_{1}$ in (83)}

By applying essentially the same arguments as Proposition 1 , we can obtain $T_{0}:=T_{B} \cdot T_{C}+T_{B}+T_{C}$ with

$$
\begin{aligned}
& T_{B}=\frac{h}{2} e^{3\|A\| h}\|A\| \cdot\left\|B_{1}\right\| c_{1} \\
& T_{C}=h\left\|C_{0}\right\| \cdot\left\|A_{2}\right\| e^{2\left\|A_{2}\right\| h} c_{\infty}
\end{aligned}
$$


while we can obtain

$$
\begin{aligned}
T_{1}: & =h\left\|C_{1}\right\| \cdot\left\|B_{1}\right\| e^{\|A\| h} \\
& +h^{2}\left\|C_{1}\right\| \cdot\left\|B_{1}\right\| \cdot\|A\| e^{2\|A\| h}\left(1+e^{\|A\| h}\right)
\end{aligned}
$$

through the arguments in [30], [31].

\section{Approximation of the Vector Set $\boldsymbol{\Phi}_{M}$}

In Section VI, we showed that the vector set $\boldsymbol{\Phi}_{M}$ can be outer-approximated by the set $\boldsymbol{\Phi}_{M}^{[N]}$ of all vectors whose image by $\Delta^{[N]}$ lies in the closed unit ball of $\mathbb{R}_{\infty}^{N n_{w}}$. In this appendix, we show that $\boldsymbol{\Phi}_{M}^{[N]}$ can be alternatively represented as the image of the closed unit ball of $\mathbb{R}_{\infty}^{N n_{w}}$ by the matrix $\Omega^{[N]}$, i.e.,

$$
\left\{w_{\Delta} \mid\left\|\Delta^{[N]} w_{\Delta}\right\| \leq 1\right\}=\left\{\Omega^{[N]} w_{d} \mid\left\|w_{d}\right\| \leq 1\right\}
$$

In the proof, we assume $n_{w}=1$ without loss of generality.

It is obvious from the definition of $v_{1}, \cdots, v_{N}$ that, for $w_{d}=[1, \cdots, 1]^{T} \in \mathbb{R}_{\infty}^{N}$, we have $\Omega^{[N]} w_{d}=[1,1]^{T}$, which coincides with the right-upper vertex of the $2 N$-polygon associated with the outer-approximation of the set $\boldsymbol{\Phi}_{M}$ (recall Fig. 3). Reversing the sign of the first entry of $w_{d}$, we readily see from the definition of $v_{1}$ that $\Omega^{[N]} w_{d}$ then coincides with the vertex of the $2 N$-polygon to the left of the aforementioned vertex. Reversing the other entries of $w_{d}$ one by one from the upper ones (until we take $N$-different values of $w_{d}$ ) and further considering the (completely) sign-reversed versions of these $N$ values of $w_{d}$, we readily see that all vertices of the $2 N$-polygon lie in the unit ball image of $\Omega^{[N]}$. Since the unit ball image is convex, we readily see that the $2 N$-polygon is contained in the unit ball image, i.e.,

$$
\left\{w_{\Delta} \mid\left\|\Delta^{[N]} w_{\Delta}\right\| \leq 1\right\} \subset\left\{\Omega^{[N]} w_{d} \mid\left\|w_{d}\right\| \leq 1\right\}
$$

To show the opposite inclusion relation, we first represent the matrix $\Delta^{[N]}$ by

$$
\Delta^{[N]}=\left[\begin{array}{c}
u_{1} \\
\vdots \\
u_{N}
\end{array}\right]
$$

where $u_{i}^{T} \in \mathbb{R}_{\infty}^{2}(i=1, \cdots, N)$. Then, we consider the matrix

$$
\Lambda^{[N]}:=\frac{1}{2}\left[\begin{array}{c}
u_{1} \\
\vdots \\
u_{N}
\end{array}\right]\left[\begin{array}{lll}
v_{1} & \cdots & v_{N}
\end{array}\right]
$$

By the definition of $u_{i}$, the $(i, i)$ entry of $\Lambda^{[N]}$ is 0 for $i=$ $1, \cdots, N$ by orthogonality. Furthermore, since all entries of the vectors $v_{1}, \cdots, v_{N}$ are non-negative and the slope $v_{i 2} / v_{i 1}$ of the $i$ th edge increases as $i$ increases, the $(i, j)$ entry of the matrix $\Lambda^{[N]}$ is positive for $i>j$ while it is negative for $i<j$. Thus, the absolute sum of the $i$ th row of $\Lambda^{[N]}$ is given by

$$
\begin{aligned}
& \frac{1}{2}\left\{\left|u_{i} v_{1}\right|+\cdots+\left|u_{i} v_{i-1}\right|+\left|u_{i} v_{i}\right|+\left|u_{i} v_{i+1}\right|+\cdots+\left|u_{i} v_{N}\right|\right\} \\
= & \frac{1}{2}\left\{-u_{i}\left(v_{1}+\cdots+v_{i}\right)+u_{i}\left(v_{i+1}+\cdots+v_{N}\right)\right\} \\
= & u_{i}\left\{\left[\begin{array}{ll}
1 & 1
\end{array}\right]^{T}-\left(v_{1}+\cdots+v_{i}\right)\right\}
\end{aligned}
$$

since $v_{1}+\cdots+v_{N}=\left[\begin{array}{ll}2 & 2\end{array}\right]^{T}$. If we note that $\left[\begin{array}{ll}1 & 1\end{array}\right]^{T}-\left(v_{1}+\right.$ $\left.\cdots+v_{i}\right)$ is nothing but the $i$ th vertex to the left from the rightupper vertex $[1,1]^{T}$ along the left-upper edges of the polygon and thus lies on the $i$ th edge of the $2 N$-polygon, we see from the definition of $u_{i}$ that the above absolute sum equals 1 for each $i=1, \cdots, N$. This implies that the unit ball image of $\Omega^{[N]}$ is contained in the $2 N$-polygon, i.e.,

$$
\left\{w_{\Delta} \mid\left\|\Delta^{[N]} w_{\Delta}\right\| \leq 1\right\} \supset\left\{\Omega^{[N]} w_{d} \mid\left\|w_{d}\right\| \leq 1\right\}
$$

This completes the proof.

\section{REFERENCES}

[1] M. Vidyasagar, Optimal rejection of persistent bounded disturbances, IEEE Trans. Automat. Contr., Vol. 31, No. 6, pp. 527-534 (1986).

[2] M. A. Dahleh and J. B. Pearson, $L^{1}$-optimal compensators for continuous-time systems, IEEE Trans. Automat. Contr., Vol. 32, No. 10, pp. 889-895 (1987).

[3] Y. Ohta, H. Maeda and S. Kodama, Rational approximation of $L_{1}$-optimal controllers for SISO systems, IEEE Trans. Automat. Contr., Vol. 37, No. 11, pp. 1683-1691 (1992).

[4] M. A. Dahleh and J. B. Pearson, $l_{1}$-optimal feedback controllers for MIMO discrete-time systems, IEEE Trans. Automat. Contr., Vol. 32, No. 4, pp. 314-322 (1987).

[5] J. S. McDonald and J. B. Pearson, $l_{1}$ optimal control of multivariable systems with output norm constraints, Automatica, Vol. 27, No. 2, pp. 317-329 (1991).

[6] I. J. Diaz-Bobillo and M. A. Dahleh, Minimization of the maximum peakto-peak gain: The general multiblock problem, IEEE Trans. Automat. Contr., Vol. 38, No. 10, pp. 1459-1482 (1993).

[7] M. Khammash, A new approach to the solution of the $l_{1}$ control problem: The scaled-Q method, IEEE Trans. Automat. Contr., Vol. 45, No. 2, pp. $180-187$ (2000).

[8] G. E. Dullerud and B. A. Francis, $L_{1}$ analysis and design of sampleddata systems, IEEE Trans. Automat. Contr., Vol. 37, No. 4 pp. 436-446 (1992).

[9] B. A. Bamieh, M. A. Dahleh and J. B. Pearson, Minimization of the $L^{\infty}$ induced norm for sampled-data systems, IEEE Trans. Automat. Contr. Vol. 38, No. 5, pp. 717-732 (1993).

[10] N. Sivashankar and P. P. Khargonekar, Induced norms for sampled-data systems, Automatica, Vol. 28, No. 6, pp. 1267-1272 (1992).

[11] J. I. Yuz and G. C. Goodwin, Sampled-data Models for Linear and Nonlinear Systems, Springer-Verlag (2013).

[12] G. C. Goodwin, J. C. Agüero, M. E. C. Garrido, M. E. Salgado and J. I. Yuz, Sampling and sampled-data models: The interface between the continuous world and digital algorithms, IEEE Contr. Syst. Mag., Vol. 33, No. 5, pp. 34-53 (2013).

[13] B. Bamieh and J. B. Pearson, The $H_{2}$ problem for sampled-data systems, Syst. Control Lett., Vol. 19, No. 1, pp. 1-12 (1992).

[14] P. P. Khargonekar and N. Sivashankar, $H_{2}$ optimal control for sampleddata systems, Syst. Control. Lett., Vol. 17, No. 6, pp. $425-436$ (1991).

[15] T. Hagiwara and M. Araki, FR-operator approach to the $H_{2}$ analysis and synthesis of sampled-data systems, IEEE Trans. Automat. Contr., Vol. 40, No. 8, pp. 1411-1421 (1995).

[16] E. N. Rosenwasser, K. Y. Polyakov and B. P. Lampe, Frequency domain method for $\mathrm{H}_{2}$-optimization of time-delayed sampled-data systems, $\mathrm{Au}$ tomatica, Vol. 33, No. 7, pp. 1387-1392 (1997).

[17] E. N. Rosenwasser and B. P. Lampe, Computer Controlled Systems: Analysis and Design with Process-orientated Models, Springer-Verlag (2000).

[18] T. Chen and B. A. Francis, Optimal Sampled-Data Control Systems, Springer-Verlag (1995).

[19] L. Mirkin, H. Rotstein and Z. J. Palmor, $H_{2}$ and $H_{\infty}$ design of sampleddata systems using lifting. Part I: General framework and solutions, SIAM J. Control and Optimization, Vol. 38, No. 1, pp. 175-196 (1999).

[20] L. Mirkin, H. Rotstein and Z. J. Palmor, $H_{2}$ and $H_{\infty}$ design of sampleddata systems using lifting. Part II: Properties of systems in the lifted domain, SIAM J. Control and Optimization, Vol. 38, No. 1, pp. 197-218 (1999).

[21] Y. Yamamoto, A function space approach to sampled data control systems and tracking problems, IEEE Trans. Automat. Contr., Vol. 39, No. 4, pp. 703-713 (1994). 
[22] B. A. Bamieh and J. B. Pearson, A general framework for linear periodic systems with application to $H^{\infty}$ sampled-data systems, IEEE Trans. Automat. Contr., Vol. 37, No. 4, pp. 418-435 (1992).

[23] H. T. Toivonen, Sampled-data control of continuous-time systems with an $H_{\infty}$ optimality criterion, Automatica, Vol. 28, No. 1 pp. 45-54 (1992).

[24] G. Tadmor, $H_{\infty}$ optimal sampled-data control in continuous time systems, Int. J. Control, Vol. 56, No. 1, pp. 99-141 (1992).

[25] T. Hagiwara and K. Okada, Modified fast-sample/fast-hold approximation and $\gamma$-independent $H_{\infty}$-discretisation for general sampled-data systems by fast-lifting, Int. J. Control, Vol. 82, No. 9, pp. 1762-1771 (2009).

[26] T. Hagiwara and H. Umeda, Modified fast-sample/fast-hold approximation for sampled-data system analysis, Eur. J. Control, Vol. 14, No. 4, pp. 286-296 (2008).

[27] G. E. Dullerud, Computing the $L_{2}$-induced norm of a compression operator, Syst. Control Lett., Vol. 7, No. 2, pp. 87-91 (1999).

[28] T. Hagiwara, Spectral analysis and singular value computations of the noncompact frequency response and compression operators in sampleddata systems, SIAM J. Control and Optimization, Vol. 41, No. 5, pp. 1350-1371 (2002).

[29] J. P. Keller and B. D. O. Anderson, A new approach to the discretization of continuous-time controllers, IEEE Trans. Automat. Contr., Vol. 37, No. 2, pp. 214-223 (1992).

[30] J. H. Kim and T. Hagiwara, $L_{\infty}$-induced norm analysis of sampled-data systems via piecewise constant and linear approximations, Automatica, Vol. 51, No. 1, pp. 223-232 (2015).

[31] J. H. Kim and T. Hagiwara, Computing the $L_{\infty}[0, h)$-induced norm of a compression operator via fast-lifting, Syst. Control Lett., Vol. 67, May, pp. 1-8 (2014).

[32] R. C. Brown and M. Tvrdý, Generalized boundary value problems with abstract side conditions and their adjoints. I, Czech. Math. J., Vol. 30, No. 1, pp. 7-27 (1980).

[33] R. C. Brown and M. Tvrdý, Generalized boundary value problems with abstract side conditions and their adjoints. II, Czech. Math. J., Vol. 31, No. 4, pp. 501-509 (1981).

[34] M. Lindner, Infinite Matrices and Their Finite Sections: An Introduction to the Limit Operator Method, Birkhäuser, Basel (2006).

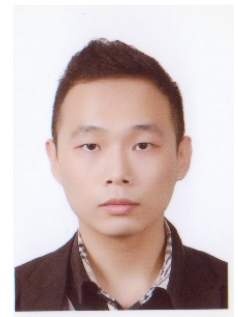

Jung Hoon Kim Jung Hoon Kim was born in Incheon, Republic of Korea, on September 22, 1988. $\mathrm{He}$ received the B. E. degree in electrical and electronic engineering, M. E. and Ph. D. degrees in electrical engineering from Kyoto University, Kyoto, Japan, in 2012, 2014 and 2015, respectively. He served as a Young Scientist Research Fellow of the Japan Society for the Promotion of Science (JSPS), DC1, from April 2014 to March 2015 and a JSPS Postdoctoral Research Fellow from April 2015 to May 2015 at Kyoto University. He is currently a Postdoctoral Researcher at the Center for Robotics Research, Korea Institute of Science and Technology (KIST), Seoul, Republic of Korea. His research interests include dynamical system theory and control applications such as $L_{1}$ optimal control, sampled-data systems, time-delay systems and Robotics.

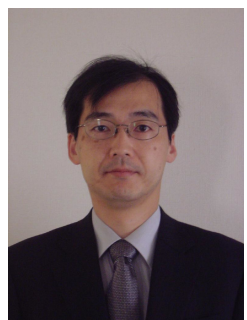

Tomomichi Hagiwara Tomomichi Hagiwara was born in Osaka, Japan, on March 28, 1962. He received the B. E., M. E. and Dr. E. (Ph. D.) degrees in electrical engineering from Kyoto University, Kyoto, Japan, in 1984, 1986 and 1990, respectively. Since 1986 he has been with the Department of Electrical Engineering, Kyoto University, where he is a Professor since 2001. His research interests include dynamical system theory and control theory such as analysis and design of sampled-data systems, timedelay systems and robust control systems. 